\title{
Objective and Subjective Effects of a Passive Exoskeleton on Overhead Work
}

\author{
Pauline Maurice, Jernej Čamernik, Daša Gorjan, Benjamin Schirrmeister, Jonas Bornmann, Luca Tagliapietra, \\ Claudia Latella, Daniele Pucci, Lars Fritzsche, Serena Ivaldi, and Jan Babič
}

\begin{abstract}
Overhead work is a frequent cause of shoulder work-related musculoskeletal disorders. Exoskeletons offering arm support have the potential to reduce shoulder strain, without requiring large scale reorganization of the workspace. Assessment of such systems however requires to take multiple factors into consideration. This paper presents a thorough in-lab assessment of PAEXO, a novel passive exoskeleton for arm support during overhead work. A list of evaluation criteria and associated performance metrics is proposed to cover both objective and subjective effects of the exoskeleton, on the user and on the task being performed. These metrics are measured during a lab study, where 12 participants perform an overhead pointing task with and without the exoskeleton, while their physical, physiological and psychological states are monitored. Results show that using PAEXO reduces shoulder physical strain as well as global physiological strain, without increasing low back strain nor degrading balance. These positive effects are achieved without degrading task performance. Importantly, participants' opinions of PAEXO are positive, in agreement with the objective measures. Thus, PAEXO seems a promising solution to help prevent shoulder injuries and diseases among overhead workers, without negatively impacting productivity.
\end{abstract}

Index Terms-Physiological measurements, workload assessment, technology acceptance, upper-limb exoskeleton.

\section{INTRODUCTION}

W ORK-related musculo-skeletal disorders (WMSDs) are the first cause of occupational diseases in developed countries [1-3]. They represent a major health issue and an important cost for companies. For instance, Fritzsche et al. showed that high ergonomic workload is related to increased absenteeism and decreased performance in automotive assembly lines [4]. WMSDs develop when biomechanical demands at work repeatedly exceed the worker's physical capacity (e.g. extreme postures, high efforts) [5]. In this regard overhead work has been identified as a major risk factor for shoulder WMSDs [6,7]. Even without external load or force exertion, supporting the arms' weight imposes prolonged stress on

Pauline Maurice and Serena Ivaldi are with Université de Lorraine, CNRS, Inria, LORIA, F-54000 Nancy, France. Jernej Camernik, Daša Gorjan and Jan Babič are with the Laboratory for Neuromechanics and Biorobotics, Department of Automation, Biocybernetics and Robotics, Jožef Stefan Institute, Jamova cesta 39, SI-1000 Ljubljana, Slovenia. Jernej Čamernik is also with the Jožef Stefan International Postgraduate School, Jamova cesta 39, 1000 Ljubljana, Slovenia. Benjamin Schirrmeister and Jonas Bornmann are with Ottobock SE \& Co. KGaA, Max-Näder Str. 15, 37115 Duderstadt, Germany. Luca Tagliapietra, Claudia Latella and Daniele Pucci are with the Fondazione Istituto Italiano di Tecnologia, via Morego 30, Genova, Italy. Lars Fritzsche is with imk automotive $\mathrm{GmbH}$, Amselgrund 30, 09128 Chemnitz, Germany.

Pauline Maurice and Jernej Čamernik contributed equally to this work.

This work has received funding from the European Union's H2020 research and innovation programme under grant agreement No. 731540 (An.Dy). shoulder muscles. Yet overhead work remains very common on assembly lines, especially in the automotive and aerospace industries. Despite growing automation, numerous strenuous tasks cannot be fully automatized, at all or at a reasonable cost. With the increase of product variants built at the same assembly line associated to small order sizes, human flexibility and cognitive skills remain needed. One solution to physically relieve workers while keeping them in control of the task execution is to assist them with an exoskeleton [8].

An exoskeleton is a wearable system that provides physical assistance to its user through assistive torques and/or structural support. Because the system is worn on the body and follows the user's movements, no, or very limited, modifications of the workplace are required [8]. Exoskeletons are therefore drawing great interest from the industry. Recently, several industrial prototypes providing arm support during overhead tasks have been developed and tested [9-19]. Some are already commercialized, e.g. the PAEXO (Ottobock, Duderstadt, Germany), the EksoVest ${ }^{\mathrm{TM}}$ (Ekso Bionics $囚$, Richmond, CA, USA), the Airframe ${ }^{\mathrm{TM}}$ (Levitate Technologies, San Diego, CA, USA), the ShoulderX (SuitX, Emeryville, CA, USA), the MATE (Comau, Turin, Italy), and the SkelEx (SkelEx, Rotterdam, The Netherlands). Most of these systems are passive, i.e. they are not powered. Instead they make use of mechanical actuation and/or springs and dampers to store and restore energy or transfer force from one part of the body to another. Passive systems have the advantage of requiring no energy source, hence being lighter than active ones, posing no autonomy issue, and presenting less safety risks for users.

Many studies on exoskeletons for overhead work showed promising results. Kim et al. [15], Van Engelhoven et al. [17], Gillette and Stephenson [12], Huysamen et al. [13] and Otten et al. [14] reported a reduction in shoulder muscle activity when performing various overhead tasks with exoskeletons (respectively EksoVest ${ }^{\mathrm{TM}}$, ShoulderX, Airframe ${ }^{\mathrm{TM}}$ and RoboMate). Butler et al. reported an increase in productivity of welders and painters equipped with the Airframe ${ }^{\mathrm{TM}}$ [11]. Spada et al. showed an increase in task performance and endurance time and a decrease in perceived discomfort, also with the Airframe ${ }^{\mathrm{TM}}$ [10]. A decrease in physical workload of the targeted limb and/or increased productivity are, however, not sufficient to demonstrate the benefit of an industrial exoskeleton. Several other factors may affect the system's effectiveness. Exoskeletons may restrict and/or modify movements kinematics $[9,16,20]$. These kinematic changes, the load transfer, or the weight of the system can increase biomechanical strain elsewhere in the body [17,20-22], and possibly affect 
postural strain $[9,16,20]$. Working with an exoskeleton may also increase physiological demand [20]. Finally, even when objective measures show positive effects, users may perceive otherwise [15] or remain dissatisfied with certain features [22].

Despite the growing number of studies on industrial exoskeletal devices and the onset of their commercialization, no standard process has been defined to thoroughly evaluate their benefit for workers $[8,15,23]$. In our view, a thorough evaluation should include objective performance measures of local effects and global effects on the user's posture, movement and effort, as well as subjective evaluations of the user's perception and acceptance of the system. Standardization of the testing procedure was initiated by Nabeshima et al., but the proposed method is specific to exoskeletons providing lumbar support and largely excludes subjective aspects [24]. Recently, Kim et al. presented an in-depth evaluation of an exoskeleton for overhead work, but physiological strain was not assessed $[15,16]$. Theurel et al. also proposed a thorough assessment

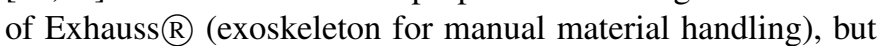
they did not test users' acceptance [20].

This work focuses on the assessment of the benefit provided by PAEXO, a novel passive exoskeleton for overhead work, which has never been quantitatively evaluated. When evaluating assistive devices such as exoskeletons, field testing with actual end-users remains the ultimate validation. However, gaining access to industrial settings and workers usually requires preliminary data to demonstrate the potential of the tested device. Besides, testing in ecological conditions restricts the number, type and invasivness of sensors used, because of environmental and/or temporal constraints (e.g., cluttered environment, extent of the workers' displacements, interferences, limited time for equipment and calibration). A preliminary lab testing is therefore needed prior to field testing. Specifically, lab testing allows an in-depth investigation of the multiple effects that an exoskeleton can cause, while field testing serves as a global validation of the device. The contribution of this paper addresses the former, i.e., we present a thorough in-lab assessment of PAEXO with data that we make publicly available. We propose an assessment method based on a large set of measures, both to evaluate effects of all kinds, and to facilitate the comparison with similar devices.

The paper is organized as follows. Section II presents the exoskeleton and the list of criteria that are evaluated. Section III describes the experimental set-up and the specific measures that implement the evaluation criteria. The results are presented in Section IV and discussed in Section V.

\section{Materials AND METHODS}

The exoskeleton evaluated in this work is the pilot series version of the commercial exoskeleton PAEXO developed by Ottobock SE \& Co. KGaA together with Volkswagen AG $[25,26]$. The testing procedure described thereafter has been developed and conducted within the EU project AnDy [27].

\section{A. Exoskeleton description}

PAEXO is a passive upper-limb exoskeleton that was designed to maximize users' freedom of movement and comfort

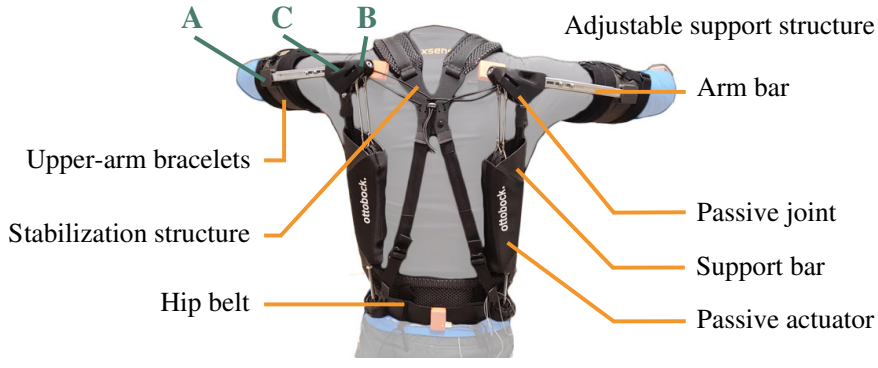

Fig. 1: PAEXO consists of a stabilization structure attached to the participant and a support structure transferring a portion of the arm weight from the upper-arm bracelets to the hip belt.

while providing a reasonable assistance. PAEXO provides a support to the user's arms by transferring a portion of the arm weight to the pelvis through a hip belt. The assistive structure of the exoskeleton consists of a support bar and an arm bar that are connected through a hinge joint (Fig. 1). A passive actuator based on a spring generates the support torque in this joint. Specifically, the arm bar is connected to the upper-arm with a bracelet -through a passive hinge joint- at one end (point $\mathrm{A}$ in Fig. 1), to a cable at the other end (point B), and to the support bar in between those 2 points (point C). The cable is attached to a spring at the lower end of the support bar. Thus, the spring applies a downward force at point $\mathrm{B}$, which generates an upward support torque in the passive joint (point $\mathrm{C}$ ). The amount of torque provided depends on the lever arm BC, which length is adjustable. The assistive structure is connected to the hip via a hip belt through a passive ball joint. The whole structure is adjustable to fit different body sizes (length of support bar, width of hip belt). A textile stabilization structure consisting of straps attached to the hip belt keeps the support structure close to the body and allows it to move comparable to the shoulder blade. The straps enable to wear the exoskeleton like a backpack. The result is a free movement of the trunk and upper extremities, with no rigid elements in the back, and for a weight of only $1.8 \mathrm{~kg}$. An analysis of PAEXO's kinematic strucutre is presented in [19], and further details on the mechanical design as well as a video demonstrating the exoskeleton are available on the product webpage https: //www.ottobock.com/en/company/ottobock-industrials/paexo/.

PAEXO is designed to generate a support torque that varies with the arm elevation angle, similarly to the torque due to the action of gravity on the arm, so that the assistance is both effective and transparent. The provided support torque is therefore maximum at an elevation angle of $90^{\circ}$ (upper arm horizontal), and is zero when the arm is lowered along the body. In addition, the level of support can be continuously tuned by adapting a mechanical parameter of the passive actuator (lever arm length BC), in order to adapt to different arm weights or compensate for the extra weight of a tool. In the prototype version used in this study, the tuning of the level of support is done manually and off-line.

\section{B. Evaluation criteria}

The primary goal of assistive exoskeletons such as PAEXO is the reduction of effort in the targeted limb. Such an 


\begin{tabular}{llll}
\hline Evaluation criteria & Performance metrics & Instrumentation & Type \\
\hline Impacted limb & Reduction of agonist muscle effort & EMG & Objective \\
\hline Side effects & No increase of effort in non-targeted muscles & EMG & Objective \\
& No reduction of postural control & Force plates for CoP movement & Objective \\
\hline Workload & Reduction of cardiovascular demand & Heart rate monitor & Objective \\
& Reduction of oxygen uptake & Oxygen consumption mask & Objective \\
\hline Task performance & No increase of movement duration & Tool motion capture & Objective \\
\hline Movement strategy & Changes in end-effector or joints trajectories & Whole-body motion capture & Objective \\
\hline Acceptance & Positive attitude towards the system & Technology acceptance questionnaire, interview & Subjective \\
& Reduction of perceived workload & Nasa Task Load Index & Subjective \\
\hline
\end{tabular}

TABLE I: Evaluation criteria, corresponding performance metrics and associated instrumentation for PAEXO assessment. The evaluation criteria cover objective and subjective effects, on the user and on the task being performed. They are defined to be generic enough to easily adapt to other exoskeletons.

improvement should, however, not be detrimental to other aspects of the users' physical, physiological or psychological well-being. We therefore propose an assessment process that addresses the following objective and subjective criteria:

- Impacted limb: The use of an exoskeleton should physically relieve the joint or limb it is designed to support.

- Side effects: The use of an exoskeleton should not increase biomechanical strain on other parts of the body.

- Workload: The use of an exoskeleton should reduce the global workload, which includes both physical and cognitive aspects. For instance, an exoskeleton that is not intuitive to manipulate might increase mental fatigue.

- Task performance: An exoskeleton should not degrade task performance or productivity. Reduced productivity has a cost for companies, and can generate stress among workers exposed to high workpace or quality pressure.

- Movement strategy: Potential modifications in users' movements due to an exoskeleton should be investigated to evaluate their consequences. For instance, disruption of natural movement may cause awkward postures or require time to learn a new motor strategy.

- Acceptance: Users should feel better, physically and mentally, when working with an exoskeleton. This is a requirement for the adoption of the system by the final end-users since an exoskeleton that is ill-perceived might remain unused, or cause psychological stress.

These six criteria constitute our basis to define the performance metrics of an exoskeleton. Each criteria is objectified by one or more performance metrics summarized in Table I and detailed in Section III-C.

\section{EXPERIMENT}

A lab study was conducted to evaluate the benefit provided by PAEXO with respect to the six criteria defined above. Participants performed a repetitive overhead pointing task, mimicking tasks commonly observed in the automotive industry, with and without the exoskeleton. The participants' physical and physiological states were monitored during the task. Participants also answered questionnaires during and after the experiment for subjective assessments. All data collected are available on-line: https://zenodo.org/record/1472214.

\section{A. Participants}

Twelve healthy males volunteered for the experiment. Their average age was $23.2 \mathrm{yrs}(\mathrm{SD}=1.2 \mathrm{yrs})$, their average stature was $179.3 \mathrm{~cm}(\mathrm{SD}=5.9 \mathrm{~cm})$, and their average body mass was $72.7 \mathrm{~kg}(\mathrm{SD}=5.4 \mathrm{~kg})$. Eight participants were right-handed and four were left-handed. All participants were college students with no or limited industrial experience and were not familiar with exoskeletons. All were naive to the purpose of the study. Participants gave written informed consent before starting the experiment. The study was approved by the Slovenian National Medical Ethics Committee (No. 339/2017/7) and was conducted in accordance to the Declaration of Helsinki.

\section{B. Experimental set-up}

1) Task description: Participants performed an overhead pointing task with a hand-held tool (power drill, weight: $0.66 \mathrm{~kg}$, tip diameter: $10 \mathrm{~mm}$ ). The goal of the task was to move the pointing tool as fast as possible from a starting point to a target, and remain on the target during $2 \mathrm{~s}$. Both the starting point and the target appeared on an interactive screen positioned horizontally above the participants' head (Fig. 2). The target turned green if hit (tool tip stopped within the target border with a $5 \mathrm{~mm}$ tolerance), and red if missed (screen touched but not on the target). If the target was missed, participants could adjust the tool position until they hit the target. Once the target was hit, a visual countdown timer informed participants how long they had to remain on the target. If the tool left the target during the $2 \mathrm{~s}$ period, the timer stopped and re-started when the tool hit the target again. At the end of the $2 \mathrm{~s}$ hold, participants freely moved the tool to the next starting position. The target was always localized in the center of the screen and appeared in 2 different sizes: $23 \mathrm{~mm}$ and $46 \mathrm{~mm}$ diameter. The starting point appeared in 4 different points equally spaced on a circle of radius $1 / 6^{\text {th }}$ of the participant's height and centered on the target.

All participants were instructed to manipulate the tool with their right hand, regardless of their handedness. They were told to keep their left hand on the frame of the screen above their head, and their feet on two signs placed on the floor below the screen. The screen height was adjusted for each participant so that the tool tip touched the screen when the participant was standing with his shoulder and elbow flexed at $90^{\circ}$ (Fig. 2). 

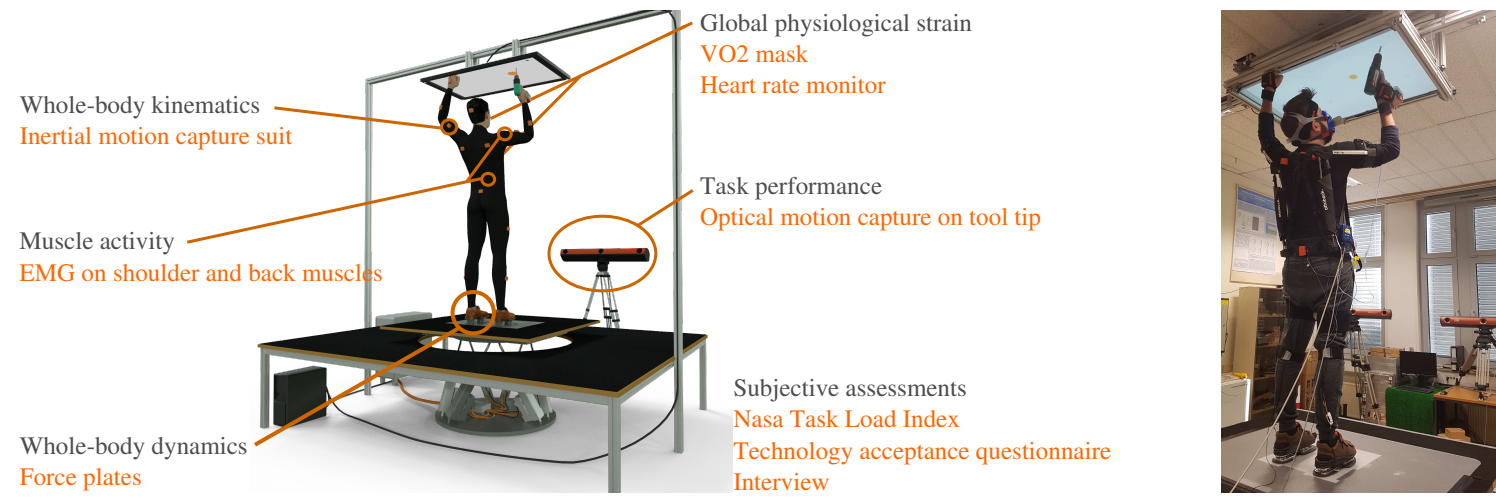

Fig. 2: Experimental set-up. Left: Schematic view of the task and sensors used to monitor the participants' physical and physiological states during the execution of the overhead task. Right: A participant performing the task with the exoskeleton.

2) Experimental design: All participants performed the task both wearing the exoskeleton (WE session), and without wearing it (NE session). A session consisted of 5 blocks of 24 pointing movements each, with a $30 \mathrm{~s}$ break between blocks. Each block lasted approximately $2 \mathrm{~min}$, resulting in a $12 \mathrm{~min}$ long session. Within a block, each combination of target size ( 2 sizes) and starting position (4 positions) appeared 3 times in random order. Participants were instructed to always keep both their arms overhead during a block. During breaks, they could lower their arms but had to keep standing. Between two sessions, participants sat and rested during $15 \mathrm{~min}$ to avoid excessive fatigue. Participants did not practice the task nor familiarize with the exoskeleton before starting the experiment.

Participants were equally divided into 2 groups: A and B. Group A started with the exoskeleton and then removed it for the second session, while Group B did the opposite to counterbalance the effects of learning and/or fatigue.

3) Exoskeleton tuning: PAEXO's structure was adapted to fit each participant's morphology. The arm bracelets were positioned at half the upper arm length. The level of support was adapted for each participant so that the arm weight was fully compensated when the shoulder and elbow were both flexed at $90^{\circ}$. This tuning was done manually by an expert before starting the experiment: the expert supported the participant's elbow to help him fully relax his shoulder muscles, then he suddenly released his support, and the movement of the participant's arm served to indicate if the tuning was correct. The tuning was considered correct if the arm did not move when the support was removed (if the arm moved downward, the assistance was too weak; if it moved upward, the assistance was too strong). This experimental tuning procedure resulted in an approximate compensation of the arm weight.

\section{Performance metrics}

The evaluation criteria listed in Section II-B define the main topics that should be assessed when evaluating an industrial exoskeleton. These criteria however need to be objectified with measures specific to the type of exoskeleton and task that are tested. Many assessment studies of industrial exoskeletons [8,20,28-30], and specifically of exoskeletons for overhead work $[10-18,21,22,31]$, are available in the literature. Therefore, suitable and validated performance metrics do exist. But exoskeleton studies have so far focused on the evaluation of a single, or a limited number, of performance criteria. Instead, we propose to evaluate the performance metrics commonly used in the literature all simultaneously, in order to obtain a thorough assessment of PAEXO with respect to all the criteria defined in Section II-B. The use of standard metrics, detailed hereafter, will facilitate the comparison of PAEXO with similar exoskeletons, both for the current and for future studies.

1) Impacted limb: The local assistive effect of an industrial exoskeleton on the targeted limb is usually quantified with a direct measure of muscle activation $[8,12-15,17,20,22,28$ 30]. For exoskeletons that aim at supporting the arm weight, the anterior deltoid $(\mathrm{AD})$ is monitored since $\mathrm{AD}$ is the primary shoulder agonist muscle involved in overhead work $[12,14,15,17,22]$. We therefore use the RMS value of AD activation to assess the physiological demand on the shoulder.

2) Side-effects: A passive exoskeleton can cause different types of biomechanical side-effects, at local and global levels. The force transfer induced by the exoskeleton and the weight of the system may create additional efforts in non-targeted muscles. Those local side-effects are usually quantified with the activation of muscles where such effects are suspected to happen [12,13,16,20-22]. For exoskeletons such as PAEXO that transfer force to the pelvis, muscles of the low back area are monitored, and among those, the erector spinae (ES) is most commonly used $[12,13,22]$. We thus use the RMS value of ES activation to assess the physiological strain in the back.

At a global level, the use of an exoskeleton may affect balance due to the additional weight of the system and/or a reduced freedom of movement. A degradation in postural control can increase the risk of fall, but also fatigue due to continuous balance adjustements. Postural control is traditionnally quantified by the displacement [20] and/or the velocity [16] of the center of pressure (CoP). Similarly, we evaluate balance-related postural strain both with the RMS of the CoP displacement with respect to its mean position (postural sway), and with the RMS of the CoP velocity.

3) Workload: Local measures such as those mentioned in the previous sections allow to understand the detailed biomechanical effects of an exoskeleton. But those measures are not sufficient since i) they do not account for possible cognitive fatigue caused by the use of the exoskeleton, and ii) unforeseen side-effects not captured by local measures might happen (all 
muscles cannot be monitored). The global physiological strain should therefore be evaluated as a complement and validation. The gold standard measure is the energy expenditure. Among standard methods for measuring energy expenditure, oxygen consumption is a good compromise between accuracy and ease-of-use [32]. It is therefore widely used, and has already been proposed for exoskeleton assessment [28,30]. Measurement of oxygen consumption however requires an invasive mask. Thus, heart rate is sometimes preferred, especially for field testing [18,20]. Though less accurate than oxygen consumption, heart rate correctly estimates energy expenditure in moderate to vigorous activities [32]. Specifically, Moyon et $a l$. reported that heart rate correctly indicates tasks perceived as strenuous by operators in a manual job including overhead work [18]. In the present lab study where sensor invasivness is not a concern, we use both the average values of oxygen consumption and heart rate to estimate the total workload. Those redundant measurements serve to validate the use of heart rate as a proxy for energy consumption in the specific case of PAEXO, in preparation for future field testing.

4) Task performance: In lab experiments, the metrics used to assess the task performance (hence the productivity) depends on the specific instructions given to the participants. Common measures are endurance time [10,11,29], number of errors [15], number of repetitions during a time period [10], and task completion time $[9,10,15,20]$. Here, participants were instructed to reach as fast as possible to the target, therefore task performance is assessed by the duration of movement in each trial, i.e. duration between the moment the tool tip leaves the start position and the end of the $2 \mathrm{~s}$ hold after target hit.

5) Movement strategy: The impact of an exoskeleton on movement strategy is commonly evaluated based on joint kinematics, using range of motion or maximal value [16,28,30], average value [20,29] (mostly for static tasks), or temporal profile [9]. The pointing task performed in the present study being non-static, we use the maximum joint angles of the right shoulder, right elbow, low back and hips to quantitatively compare the two conditions WE/NE. Different movement strategies can however lead to a same range of motion. We therefore also analyze the temporal profiles of those same joint angles to qualitatively identify any kinematic changes. In addition, since unconstrained reaching motions are known to exhibit a very typical kinematic pattern (minimun-jerk trajectory [33]), we monitor the Cartesian trajectory of the tool to verify whether the exoskeleton disrupts this pattern.

6) Acceptance: In addition to objective measures, exoskeleton studies often include subjective measures to evaluate the agreement between the physiological and perceived effects. Borg perceived exertion or discomfort scale [34] is largely used [10,13-15,18,20,22], but it only focuses on the physical aspect. We rather use the Nasa Task Load Index (Nasa-TLX) to assess the global perceived workload [35]. The Nasa-TLX is a combined measure taking into account 6 factors - mental demand, physical demand, temporal demand, performance, effort and frustration - each assessed on a 20-point scale. A global score is calculated by weighing each factor with task and participant-specific weights. Though Nasa-TLX has not been previously used for exoskeleton evaluation, it is a validated measure which covers physical and cognitive aspects.

Acceptance of an exoskeleton is however affected not only by the perceived workload, but also by other factors, such as usability, comfort, or image. Quantitative assessment of users' opinions and attitudes towards an exoskeleton usually relies on standard technology acceptance questionnaires [10,13], such as the Technology Acceptance Model (TAM3) [36], the Unified Theory of Acceptance and Use of Technology (UTAUT) [37], or the System Usability Scale (SUS) [38]. We created an ad-hoc questionnaire based on the TAM3 and UTAUT models (available in the supplementary material), since those models are not limited to usability, and are therefore more exhaustive. Nevertheless, we focused most of the questions on comfort and usability aspects to detect potential design issues, and we excluded constructs such as image or job relevance since the participants in the present study were not actual end-users of the system (those topics would have to be added for field testing). The questionnaire thus consists of 20 questions each assessed with a 10-point Likert scale and covering 8 constructs: perceived usefulness, perceived ease of use, intention of use, facilitating condition, attitude towards the system, trust, enjoyment and ethics. A global acceptance score is computed as the average across all questions. Separate scores for each of the 8 constructs are also computed for a detailed analysis (average across all questions in each construct). In addition, similarly to Spada et al. [10], we conducted a semi-directed interview to clarify and complement the questionnaire answers by allowing participants to express more detailed opinions (outline available in the supplementary material).

\section{Instrumentation}

This section describes the sensors and procedures used to measure the above-mentioned performance metrics.

1) Tool kinematics: The tool 3D trajectory was tracked with the NDI 3D Investigator optical motion capture system using 3 markers attached to the tool (NDI, Waterloo, Canada). Data were recorded at $100 \mathrm{~Hz}$ with a Matlab script running on Simulink Real-Time.

2) Whole-body kinematics: Participants were equipped with an Xsens inertial motion tracking suit consisting of 17 IMUs to record whole-body kinematics (Xsens, Enschede, The Netherlands). Data were recorded with the Xsens MVN software at $60 \mathrm{~Hz}$. The system was calibrated at the beginning of each session following the Xsens MVN calibration procedure.

3) Ground reaction force: Participants stood with each foot on a Kistler force plate to measure the CoP trajectory (Kistler, Winterthur, Switzerland). Data were recorded at $1000 \mathrm{~Hz}$ with a Matlab script running on Simulink Real-Time.

4) Surface electromyography (EMG): Muscle activity of the right anterior deltoid (AD) and right erector spinae longissimus (ES) was recorded with the Biometrics DataLOG MW8X EMG system using the SX230 sensor (Biometrics Ltd, Newport, UK). Right side muscles were monitored since participants performed the task with their right hand. Electrodes were placed on the skin following SENIAM recommendations [39]. EMG signals were recorded at $1000 \mathrm{~Hz}$ with a Matlab script running on Simulink Real-Time. After recording, EMG 
signals were high-pass filtered ( $4^{\text {th }}$ order Butterworth filter with a $20 \mathrm{~Hz}$ cut-off frequency), low-pass filtered ( $4^{\text {th }}$ order Butterworth filter with a $450 \mathrm{~Hz}$ cut-off frequency), detrended and rectified. The envelop of the signal was then extracted using a low-pass filter ( $4^{\text {th }}$ order, $10 \mathrm{~Hz}$ cut-off frequency). Finally, the signal was normalized by the maximum value reached during the task (both NE and WE) for each participant.

5) Heart rate: A Polar WearLink+ Transmitter with Bluetooth heart rate sensor was placed on participants' chest to monitor their heart rate (Polar Electro Inc., Bethpage, USA). Data were recorded using a mobile application provided with the sensor. Heart rate was then normalized using maximum and minimum values of the participant in the NE condition.

6) Oxygen consumption: Participants were equipped with a VO2 Master Pro mask to measure their oxygen consumption (VO2 Master Health Sensors Inc., Vernon, Canada). The sensor was calibrated before each session. Data were recorded with a mobile application provided with the sensor. Oxygen consumption was normalized by the participant's weight.

7) Perceived workload: The 6 factors of the Nasa-TLX questionnaire were evaluated by the participants after each session (i.e., once for WE and once for NE). Conversely, the weights of each factor were selected only once at the end of the experiment so that they were the same for the 2 conditions.

8) Technology acceptance: The technology acceptance questionnaire was filled once by each participant at the end of the experiment, and the interview was conducted afterward.

\section{E. Statistical analysis}

The two sessions WE and NE are compared to assess the effect of the exoskeleton (except for the acceptance questionnaire). The evolution of the performance metrics over time (i.e., across the 5 blocks of a session) is also analyzed to detect effects of learning and fatigue. Statistical analyses therefore test the effect of two factors: intervention (with or without exoskeleton) and block number. Data are checked for normality with the Shapiro-Wilk test. A significance level of $5 \%$ is adopted for all statistical tests, and analyses are performed with R. Oxygen consumption, heart rate and Nasa-TLX match the normality assumption, whereas all other variables do not.

Data that follow a normal distribution are analyzed with a two-way repeated-measures analysis of variance (ANOVA), with two within-subject fixed factors: intervention and block number. Participants are entered as a random factor. Pairwise multiple comparison post-hoc tests with Bonferroni corrections are conducted for each fixed factor which has a significant effect according to the ANOVA. Data that violate the normality assumption are analyzed with separate tests for each factor. The effect of intervention is assessed with a Wilcoxon signed-rank test. The average value of the dependent variable across all 5 blocks is used to obtain one single measure per intervention (WE/NE) for each participant. A Friedman test is performed separately for the WE and NE conditions to assess the effect of block number. Pairwise multiple comparison posthoc tests with Bonferroni corrections are conducted when the block factor has a significant effect. The Nasa-TLX was not measured for each block but for a full session (WE/NE), so it is analyzed with a paired-t-test.

\section{RESUlTS}

The evaluation of the six criteria defined in section II-B are presented hereafter, using the metrics of Section III-C.

\section{A. Impacted limb}

Fig. 3a displays the evolution of $\mathrm{AD}$ activation across blocks for all participants, for the two interventions WE and NE. Using the exoskeleton (WE) leads to a significant reduction in $\mathrm{AD}$ activation compared to the non-assisted condition (NE) $(p<.001)$. With the exoskeleton, AD activation is smaller by $54 \%$ in average. The effect of the block number is not significant when using the exoskeleton $(p=.15)$, whereas it is significant when performing the task without assistance $(p<.001)$. Without assistance, AD activation increases by $30 \%$ in average between the first and last blocks.

\section{B. Side effects}

Fig. 3b displays the evolution of ES activation across blocks for all participants, for the two interventions WE and NE. No significant difference in ES activation is observed between the two interventions $(p=.70)$. The block number factor has no significant effect on ES activation when wearing the exoskeleton $(p=.07)$, nor when not wearing it $(p=.41)$.

Figs. $3 \mathrm{c}$ and $3 \mathrm{~d}$ display the evolution across blocks of the $\mathrm{CoP}$ displacement and $\mathrm{CoP}$ velocity for all participants, for the two interventions WE and NE. The use of the exoskeleton marginally affects the CoP displacement $(p=.064)$, while it significantly affects the CoP velocity $(p=.007)$. With the exoskeleton, the CoP displacement is smaller by $18 \%$ in average and the CoP velocity by $14 \%$ compared to the non-assisted condition. The block number factor significantly affects the CoP displacement, both with the exoskeleton $(p=.036)$ and without it $(p<.001)$. The CoP displacement increases by $30 \%$ in average between the first and last blocks when the exoskeleton is used, while the increase reaches $50 \%$ without the exoskeleton. Conversely, the effect of the block number factor on the CoP velocity is significant in the non-assisted condition $(p=.011)$, but not when using the exoskeleton $(p=.18)$. In the non-assisted condition, the CoP velocity increases by $13 \%$ in average between the first and last blocks.

\section{Workload}

Fig. 3e and $3 f$ display the evolution across blocks of oxygen consumption and heart rate for all participants, for the two interventions WE and NE. Both oxygen consumption and heart rate are significantly affected by the exoskeleton (oxygen consumption: $F(1,11)=17.43, p=.0016, \eta_{G}^{2}=.11$; heart rate: $\left.F(1,11)=7.08, p=.022, \eta_{G}^{2}=.13\right)$. With the exoskeleton, oxygen consumption is lower by $33 \%(p<.001)$ and heart rate by $19 \%(p<.001)$ in average.

The ANOVA reveals a significant effect of block number on both variables (oxygen consumption: $F(4,44)=12.24$, $p<.001, \eta_{G}^{2}=.12$; heart rate: $F(4,44)=43.41, p<.001$, $\left.\eta_{G}^{2}=.26\right)$. Oxygen consumption increases significantly between the first and last blocks $(p<.001)$, and so does heart rate $(p<.001)$. The ANOVA reveals an interaction 


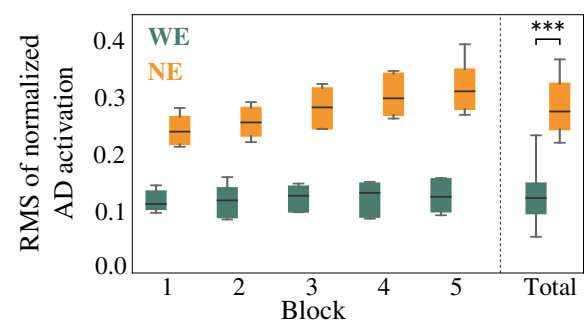

(a) Anterior Deltoid (AD) activation

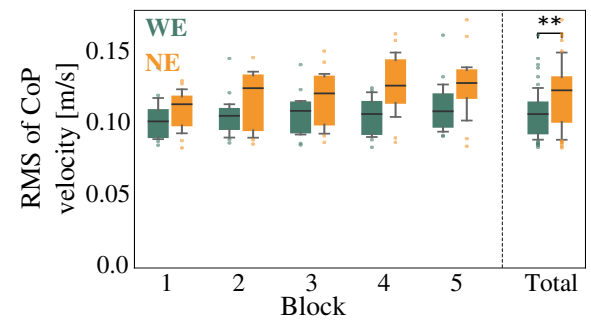

(d) CoP velocity

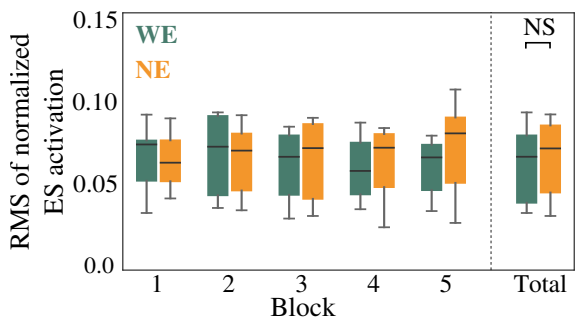

(b) Erector Spinae (ES) activation

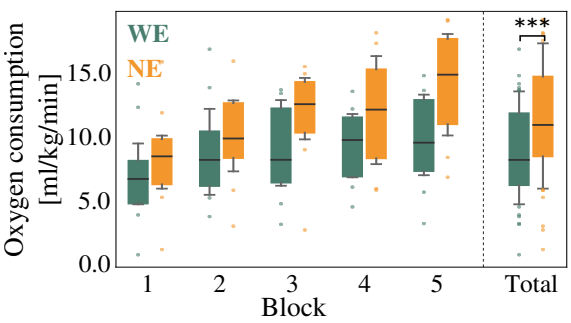

(e) Oxygen consumption

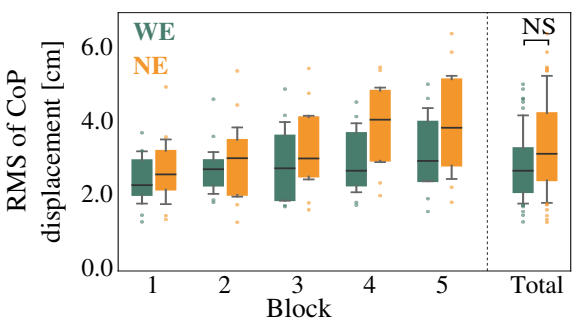

(c) CoP displacement

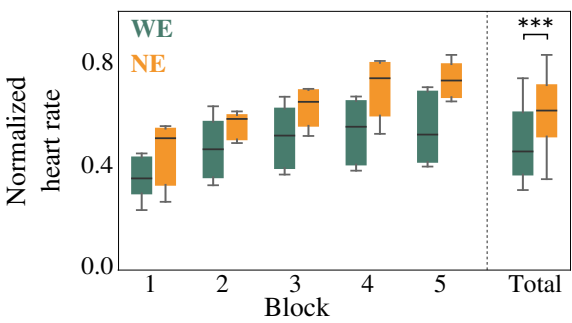

(f) Normalized heart rate

Fig. 3: Evolution of impacted limb (a), side effects (b,c,d) and workload (e,f) objective metrics across blocks for all participants, for the two interventions, WE and NE. The box plots are created using all trials of all participants. NS stands for not significant.

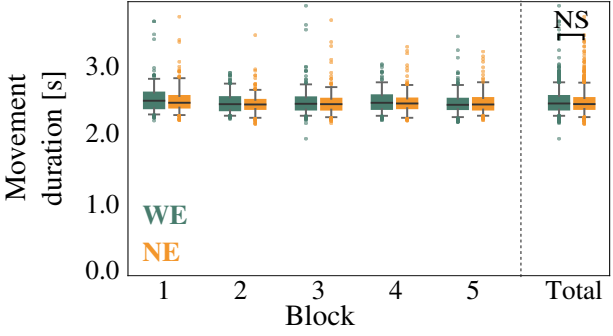

Fig. 4: Evolution of movement duration across blocks for all participants, for the two interventions, WE and NE.

between the intervention and block number factors for heart rate $\left(F(4,44)=2.99, p=.029, \eta_{G}^{2}=.021\right)$, but not for oxygen consumption $\left(F(4,44)=1.79, p=.15, \eta_{G}^{2}=.018\right)$. Heart rate increases by $56 \%$ in average between the first and last blocks when using the exoskeleton, and by $65 \%$ without it. Though no significant interaction between factors is detected, oxygen consumption increases by $37 \%$ between the first and last blocks when using the exoskeleton, whereas the increase reaches $65 \%$ in the non-assisted condition.

\section{Task performance}

Fig. 4 displays the evolution across blocks of movement duration for all participants, for the two interventions WE and NE. No significant difference in movement duration is observed between the two interventions $(p=.38)$. The effect of the block number factor is significant with the exoskeleton $(p=.017)$ and marginally significant in the non-assisted condition $(p=.058)$. With the exoskeleton, movement duration decreases by $3 \%$ in average between the first and last blocks.

\section{E. Movement strategy}

The tool and joint trajectories necessarily differ according to the movement start position and possibly to the target size (the experimental task included 4 different start positions and
2 target sizes, see Section III-B1). Trajectories and maximum joint angles are therefore analyzed separately for each of the 8 combinations of start positions and target sizes (only the start-to-target and hold motions are included, the return motion is excluded). For the sake of simplicity, graphs are only displayed for one of the 8 aforementioned combinations: small target size and start position located on the left front side of the participants. Trajectories of all trials are time-scaled to a common duration to enable the computation of an average profile. Graphs for the other start positions and target sizes are available in the supplementary material.

a) Tool movement: Fig. 5a displays the trajectory and velocity of the tool tip for all participants, for both interventions WE and NE. The movement of the tool tip with the exoskeleton closely resembles the movement without the exoskeleton. Specifically, the variability between conditions (i.e. WE vs. NE) is not bigger than within one condition.

b) Arm movement: Fig. 5b displays the time-series of the shoulder and elbow joint angles of all participants for both interventions WE and NE. Shoulder joint movements differ between both interventions. Maximum shoulder abduction is significantly or marginally affected by the use of the exoskeleton for all start positions and all target sizes (p-value varies between .003 and .092). Arm abduction is larger with the exoskeleton, by $7.9 \mathrm{deg}$ to $9.9 \mathrm{deg}$, depending on the target size and start position. Conversely, maximum shoulder flexion and rotation are significantly affected by the exoskeleton only for the start position located on the right front side of the participants. For this start position, shoulder flexion is larger with the exoskeleton by $9.4 \mathrm{deg}$ in average for the small target $(p=.007)$ and by $9.5 \mathrm{deg}$ for the large target $(p=.009)$, while shoulder rotation is larger with the exoskeleton by $9.2 \mathrm{deg}$ for the small target $(p=.009)$ and by $6.9 \mathrm{deg}$ for the large one $(p=.007)$. Maximum elbow flexion is significantly affected by the exoskeleton only for the start position located on the left front side of the participants. The biomechanical difference 

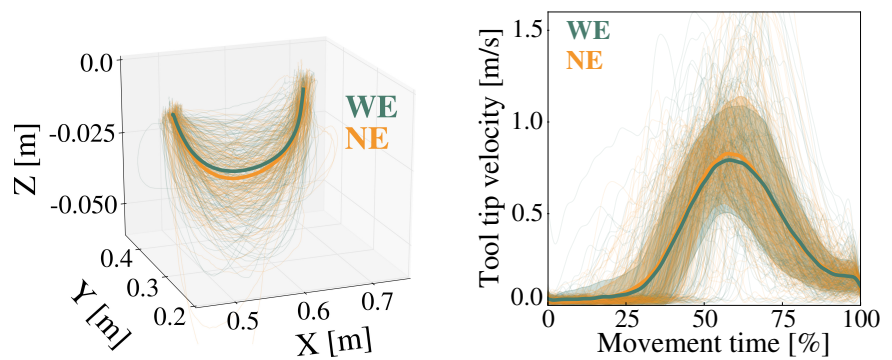

(a) Left: 3D trajectory of the tool tip. Right: time-series of the tool tip velocity. The trajectories and time-series represent only the reaching phase, i.e. they start when the tool tip leaves the start position ( $0 \%$ of movement time) and end at first target hit (100\% of movement time); the $2 \mathrm{~s}$ hold phase is not included.
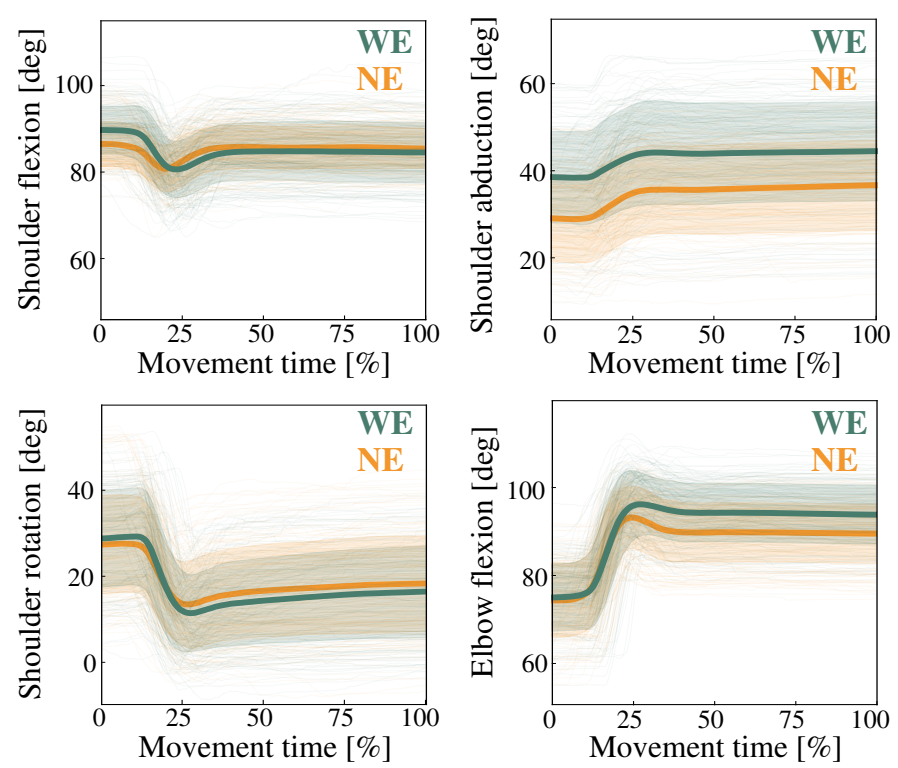

(b) Time-series of the right shoulder and elbow joints. The time-series represent the movement from when the tool tip leaves the start position $(0 \%$ of movement time) to the end of the $2 \mathrm{~s}$ hold after target hit ( $100 \%$ of movement time).

Fig. 5: Movement of the tool tip (a) and of the arm (b) for all participants and all blocks, for the two interventions WE and NE (trials corresponding to the small target size and the start position located on the left front side of the participants). Thin lines, bold lines and shaded areas represent respectively individual trials, average, and standard deviation profiles.

between the two conditions WE and NE is however small: with the exoskeleton, the maximum elbow flexion is larger by $4.9 \mathrm{deg}$ in average for the small target $(p=.003)$ and by $3.2 \mathrm{deg}$ for the large target $(p=.02)$.

c) Back and hip movement: Neither back maximal extension nor back maximal lateral bending are significantly affected by the use of the exoskeleton ( $\mathrm{p}$-value varies between .30 and 1.0 for back extension and between .62 and 1.0 for lateral bending, depending on the target size and start position). Conversely, torso rotation is significantly affected by the use of the exoskeleton (p-value varies between .002 and .052 depending on the target size and start position). Torso maximal rotation is smaller by $1.3 \mathrm{deg}$ to $3.6 \mathrm{deg}$ (depending on the target size and start position) with the exoskeleton. During the whole experiment (excluding rest periods), hips were slightly flexed, i.e., the pelvis was inclined backwards. Hip extension is, however, not affected by the use of the

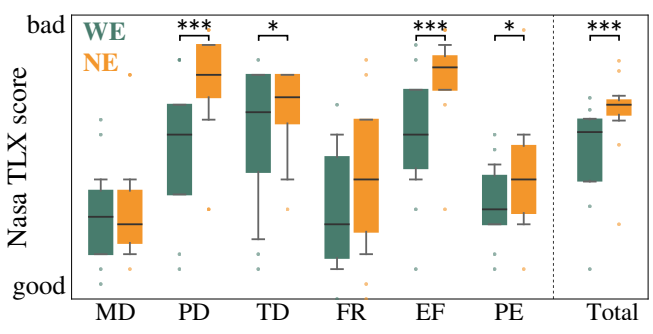

Fig. 6: Nasa-TLX scores of all participants, for the two interventions NE and WE. Scores are presented for the 6 factors separately (mental demand: MD; physical demand: PD; temporal demand: TD; frustration: FR; effort: E; performance: $\mathrm{PE})$, and globally (weighted sum of scores of each factors).

\begin{tabular}{lllllllll}
\hline Construct & PU & PEOU & IU & FC & ATTD & TR & EN & ET \\
\hline Mean & 7.40 & 7.54 & 8.83 & 8.67 & 8.19 & 8.08 & 6.58 & 7.00 \\
\hline SD & 1.82 & 1.84 & 2.37 & 1.50 & 1.78 & 1.20 & 1.44 & 1.71 \\
\hline
\end{tabular}

TABLE II: Mean and standard deviation of the technology acceptance scores for the 8 constructs covered by the questionnaire (PU: perceived usefulness; PEOU: perceived ease of use; IU: intention of use; FC: facilitating condition; ATTD: attitude towards the system; TR: trust; EN: enjoyment; ET: ethics). Scores can vary between 0 (bad) and 10 (good).

exoskeleton (p-value varies between .27 and .85 depending on the target size and start position). The profiles of the back and hip joint angles are plotted in the supplementary material.

\section{F. Acceptance}

Fig. 6 presents the global and detailed (for the 6 factors) scores of the Nasa-TLX for all participants, both with and without the exoskeleton (the numerical scores of the NasaTLX are reported in the supplementary material). The global Nasa-TLX score is significantly lower, by $21 \%$ in average, when using the exoskeleton compared to the non-assisted condition $(p<.001)$. The difference WE $v s$. NE is significant for the physical demand $(p<.001)$, temporal demand $(p=.034)$, performance $(p=.024)$ and effort $(p<.001)$ factors. For all those factors the score is lower with the exoskeleton, but the magnitude of the difference is the biggest for the two forcerelated factors: physical demand and effort.

Table II displays the technology acceptance scores of the 8 constructs covered in the questionnaire, for all participants. The global score, computed for each participant as the average of the scores of the 8 constructs, is $7.76(\mathrm{SD}=1.79)$, with 0 corresponding to no acceptance at all and 10 to full acceptance.

In the interviews, all participants said they found the system helpful to decrease physical fatigue. When asked about freedom and intuitiveness of movement with the exoskeleton, participants answered that they did not feel restrained in their movements, except in some extreme postures (shoulder retractation and full shoulder flexion, which were not needed to perform the task). In addition, they reported that they had to get used to working with the exoskeleton in the first few movements, but that it was not cognitively demanding. No participant expressed any fear or concern about wearing the 
exoskeleton. Regarding comfort, participants said the weight of the system was satisfying, but several of them found the arm bracelets of this prototype uncomfortable. Importantly, all participants answered positively when asked if they would want to use the exoskeleton again in a similar task.

\section{Discussion}

In this study, twelve non-expert participants performed an overhead pointing task with and without the assistance of PAEXO, in order to evaluate the benefit provided by the exoskeleton. Six criteria were assessed: loading of the impacted limb, side-effects, global workload, task performance, movement strategy and user acceptance. The results are overall positive and are discussed hereafter.

\section{A. Reduction of strain and fatigue}

Our results show that PAEXO alleviates physical strain on the shoulder during overhead work: AD activation is reduced by $55 \%$. This value is within the range reported for similar exoskeletons: $27 \%$ to $58 \%$ reduction in $\mathrm{AD}$ activation with the passive setting of Lucy 2.0 [14], $24 \%$ to $80 \%$ with the ShoulderX [17], $28 \%$ in average with the EksoVest ${ }^{\mathrm{TM}}$ [15], and around $20 \%$ with the Airframe ${ }^{\mathrm{TM}}$ [12]. Though they did not monitor AD, Huysamen et al. observed up to $50 \%$ reduction in shoulder muscle activity with the Robo-Mate [13].

Reduced shoulder strain is positive. Yet passive systems do not input energy, so the assistive force they provide has to be induced back to another, generally stronger, part of the body [8]: the pelvis in the case of PAEXO. Despite this force transfer, the similarity of ES activation measured with and without the exoskeleton suggests that PAEXO does not increase strain in the surrounding low back area. Nonsignificant differences in back muscle activity with and without exoskeleton were also reported with the Robot-Mate [13] and with the Airframe ${ }^{\mathrm{TM}}$ [12], while the EksoVest ${ }^{\mathrm{TM}}$ caused mixed effects on spine loading [16].

At the global level, the effect of PAEXO is also beneficial. The reduction and reduced increase over time of both oxygen consumption and heart rate show that physiological strain is lower when using PAEXO, which suggests that the task is overall less fatiguing with the exoskeleton (in addition, the similar trends in oxygen consuption and heart rate suggest that heart rate is a reasonable proxy to evaluate energy expenditure with PAEXO when sensor invasivness is an issue). Moyon et al. similarly observed a reduction in heart rate when using SkelEx for an overhead sanding task [18]. Conversely, Theurel et al. reported a negative effect of Exhauss $(\mathrm{B}$ on heart rate during a lifting task [20]. This opposed trend is possibly due

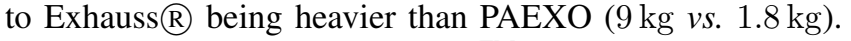

Eventually, unlike the EksoVest ${ }^{\mathrm{TM}}$ [16] and Exhauss $\mathbb{R}$ [20], PAEXO did not degrade postural control. This difference may again be explained by the light weight and flexibility of PAEXO's structure compared to other systems (EksoVest $^{\mathrm{TM}}$ : $6.5 \mathrm{~kg}$ for the version in [16], $4.3 \mathrm{~kg}$ for the current commercial version). Conversely both $\mathrm{CoP}$ displacement and velocity are decreased with PAEXO. This reduction is not due to overall slower movements since movement duration is not affected. Instead, the increase in CoP displacement and velocity over time suggests that changes in postural control reflect balance perturbations due to fatigue. The smaller and smoother movements of the CoP when using PAEXO may therefore be an additional indicator showing that PAEXO reduces fatigue.

\section{B. Productivity}

The similarity of movement duration with and without the exoskeleton shows that wearing PAEXO does not affect task performance positively or negatively. The absence of significant changes in movement duration over time suggests that no extended practice is necessary to use PAEXO. This finding is confirmed by participants' report that getting used to working with the system was very fast.

Though PAEXO does not slow down the movement, our results do not indicate that it increases productivity either. Conversely, Kim et al. observed a $19 \%$ reduction in movement completion time in an overhead drilling task when using the EksoVest $^{\mathrm{TM}}$ [15]. A major difference between Kim's task and ours is the amount of effort involved, their task being 6 times longer and with a $110 \mathrm{~N}$ force exertion. Physical fatigue tends to slow down movements and cause a loss of motion control. The effect of exoskoskeletal assistance in preventing workpace reduction over time is therefore more likely to be observed when the task is longer and/or more strenuous. For instance, two separate studies reported that the Airframe $^{\mathrm{TM}}$ increases endurance time and hence productivity over prolonged period of time [10,11]. In our study, the short duration of a session did not allow fatigue to develop enough to degrade movement speed or accuracy. Nevertheless, since PAEXO reduces physiological strain (Section V-A), hence likely the development of fatigue, it is expected that using PAEXO will increase the productivity in longer tasks, though this hypothesis needs to be confirmed by further investigations.

\section{Movement strategy}

Using PAEXO does not impact the Cartesian trajectory of the tool tip. In particular, the bell-shape profile of the endeffector velocity typical in reaching movements is retained (Fig. 5a) [33]. At joint level, however, arm movement differs with and without the exoskeleton. When wearing PAEXO, the elbow is elevated on the side of the body, i.e. the upper arm is abducted, whereas arm abduction is smaller without the exoskeleton. In a study on the Airframe ${ }^{\mathrm{TM}}$, participants reported that they had to fight the system to maintain an adducted arm posture [10]. Conversely, participants in our study reported that during the task their movements felt natural and unrestricted when wearing PAEXO. They also gave the question "do you feel free in your movements when using the exoskeleton?" an average score of 6.9 (SD $=1.8$, where $0=$ not free at all, and $10=$ totally free). Therefore the difference observed in shoulder movement with and without the exoskeleton is likely not caused by movement hindrance due to the system. Instead, participants may have deliberately chosen a different movement strategy when wearing PAEXO. The RULA ergonomic assessment worksheet handles upper arm abduction as a penalizing factor, thus suggesting that 
maintaining an abducted arm posture is demanding for shoulder muscles [40]. Participants may therefore have naturally avoided such a posture when working without assistance. Conversely when using PAEXO, a large part of the arm weight is compensated by the system. Adopting a posture which minimizes demand on shoulder muscles may then be less critical, and participants may have prioritized other criteria to choose their preferred posture [31].

\section{Users' opinion}

Objective measures show that using PAEXO reduces physical and physiological strain (Section V-A). Results of the subjective Nasa-TLX assessment indicate that also the perceived workload is reduced with PAEXO, and by the same order of magnitude as the reduction in global physiological strain $(21 \%$ reduction for Nasa-TLX vs. $19 \%$ for heart rate and $33 \%$ for oxygen consumption). Those results are aligned with the high score of the question "I feel less tired when using the exoskeleton" ( $7.3 \pm 1.0)$. The high score of perceived usefulness and intention to use constructs in the technology acceptance questionnaire (Table II) and the will expressed by all participants to use the exoskeleton again also reflect a positive opinion towards the system. At individual factors level, the significant reduction in the physical demand and effort factors of the Nasa-TLX with PAEXO, along with the high scores of the questions "The exoskeleton is helpful during the gestures" (8.2 \pm 1.1$)$ and "The exoskeleton helps me doing my movement in an ergonomic manner" (7.9 \pm 1.1$)$, qualitatively match the measured reduction in shoulder physical strain. In agreement with the measured absence of negative side-effects, participants reported that using PAEXO did not require specific physical nor cognitive efforts (the question "The exoskeleton requires some physical (resp. cognitive) efforts" received a score of $6.8 \pm 1.9$ (resp. $7.8 \pm 1.6$ ), while the mental demand score of the Nasa-TLX remained similar with and without exoskeleton). Note that the slightly lower score of the physical effort question, compared to the cognitive effort question, might be due to the fact that PAEXO did not fully suppress all physical efforts required to perform the task: the weight of the tool was not compensated and participants still had to voluntarily move their arm. As for movement strategy, the above average scores of the questions "I feel free in my movements when I am wearing the exoskeleton" (6.9 \pm 1.8$)$ and "The exoskeleton is a constraint" $(6.7 \pm 2.4)$ agree with the moslty unchanged joint kinematics, and suggest that the change in shoulder abduction was voluntary. Finally, regarding task performance, the similarity in movement duration with and without PAEXO agrees with the high score of the question "The exoskeleton does not make me waste time" (8.8 \pm 1.3$)$. According to the performance Nasa-TLX factor, participants even perceived a slight improvement in task performance with PAEXO, which is however not supported by the objective measure. Conversely, the high scores of the questions "You don't need a long training to use the exoskeleton" (8.7 \pm 1.5$)$ and "The exoskeleton is easy to use" $(8.8 \pm 1.5)$ are well aligned with the absence of learning phenomenon in the objective task performance measure. Thus, objective phenomena and their perceptive counterparts largely match. The small differences observed might be explained by the fact that there is no oneto-one mapping between objective measures and subjective questions, the latter relating to several objective phenomena which respective influence cannot be disentangled by the question only. In addition, if usability constructs (perceived usefulness, perceived ease-of-use, facilitating condition) can be interpreted in the light of objective measures, such relation is less straightforward for image and affect-related constructs (trust, enjoyment, attitude towards the system), which more strongly depends on the personality and experience of the user.

Though concordance between objective and subjective measures has been reported with other exoskeletons [10], the biomechanical advantage provided by an assistive device is not always perceived as such by users. For instance, Kim et al. reported a disagreement between reduced shoulder muscle activity and absence of change in shoulder perceived discomfort with the EksoVest ${ }^{\mathrm{TM}}$ [15]. Yet, agreement between objective and subjective measures is crucial for a system which primary goal is the improvement of workers wellbeing, because not only physical demand, but also mental state impacts the development of WMSDs $[1,8]$.

Comfort is another important aspect for users' acceptance since PAEXO is intended to be worn for extended periods of time. Several studies on exoskeletons reported perceived discomfort due to design issues [8,21,22,29]. Conversely, participants in our study were mostly satisfied with the comfort of PAEXO: the question on comfort in the technology acceptance questionnaire got an average score of $6.8(\mathrm{SD}=2.0$, where $0=$ very uncomfortable and $10=$ very comfortable). Nevertheless, 7 out of 12 participants mentioned that the arm bracelets of the prototype were constricting their arms. Following this report, the design of the arm bracelets has been improved before commercialization. The experiment, however, does not enable to conclude whether arm bracelets were a real issue: the upper arm sensors of the motion capture system were placed below the arm bracelets and may also have caused the discomfort.

\section{E. Comparison with competing exoskeletons}

The results discussed in the previous sections suggest that PAEXO is a promising solution to reduce strain and possibly help decrease the prevalence of upper-limb WMSDs among overhead workers. Yet, other passive exoskeletons for arm support do exist (e.g., EksoVest ${ }^{\mathrm{TM}}$, Airframe ${ }^{\mathrm{TM}}$, SkelEx, MATE, ShoulderX). Those competing devices are based on similar mechanical principles to PAEXO, but their designs differ [19]. A reduction in shoulder physical strain was reported with all those exosleketons, but numerical values largely vary (Section V-A). More importantly, most of them were reported to cause some negative effects that were not observed with PAEXO (in postural control, low back strain, freedom of movement, or perceived usability $[10,13,16])$. Design differences might explain these variations from one system to another. As mentioned in Section V-A, the light weight of PAEXO compared to its concurrents is a significant advantage which certainly limits the overloading of the low back (PAEXO: $1.8 \mathrm{~kg}$; EksoVest ${ }^{\mathrm{TM}}$ : $4.3 \mathrm{~kg}$; ShoulderX: $5.3 \mathrm{~kg}$; 
MATE: $4.0 \mathrm{~kg}$; SkelEx: $3.4 \mathrm{~kg}$; Airframe $^{\mathrm{TM}}: 2.7 \mathrm{~kg}$, RoboMate: $7.2 \mathrm{~kg}[19,41])$. Nevertheless, some heavier exoskeletons offer more options as a trade-off. For instance, the MATE includes a system to modify the level of support with a button [41], whereas tools are required to adjust PAEXO's support. A second advantage of PAEXO is the flexibility of its structure: PAEXO has no rigid elements in the back, which enables the full range of upper-limb and back movements. Accordingly, participants reported that PAEXO was not constraining or limiting their movements, though the extent of the range of motion was not measured. Conversely, a $10 \%$ reduction in the shoulder range of motion was observed with the less flexible EksoVest $^{\mathrm{TM}}$ [16]. The usability study of the Robo-Mate, in which half the participants found the device too cumbersome [13], also emphasizes that a light and flexible structure such as PAEXO's is an important asset for an industrial exoskeleton. Finally, PAEXO does not generate any upward torque when the arm is lowered, unlike some competing devices (in [10] participants reported they had to fight the AirFrame ${ }^{\mathrm{TM}}$ to maintain the arm adducted). This feature enables a comfortable posture when not working overhead, without overloading the antagonist shoulder muscles.

The differences in the results observed between PAEXO and its concurrents may, however, also be partly explained by the variety of experimental tasks (static/dynamic, work height, weight of tool...) $[12,13,15,17]$, as well as by the different, and often empirical, tunings of the level of support [17]. The lack of a standard assessment procedure prevents quantitative comparison of different studies. Concluding on the better or worse performance of PAEXO compared to other exoskeletons is therefore not straightforward, despite its indisputable advantages of weight and flexibility.

\section{F. Limitations of the study}

Our results suggest that using PAEXO during overhead work is beneficial. Nevertheless this preliminary study presents some intrinsic limitations. Most importantly, participants were college students and not real end-users of the system. Aside from the lack of diversity in participants' morphology, biomechanical effects may be different for industrial workers because experts often perform technical gestures differently from novices. In addition, even though participants were instructed to move as fast as possible, the temporal pressure was not the same as what workers on assembly lines are exposed to. Small movement disturbances that the exoskeleton might cause may not be noticeable by novices under little pressure, but they may become an issue in a situation with high temporal demand. Another limitation due to the population tested is that answers to the acceptance questionnaire are likely audiencedependent. Real end-users may, for instance, be concerned by the judgment of colleagues, hierarchy or family, were they to use or not use an exoskeleton [42]. Thus, the present results should be considered carefully. This study is however not intended to be the only one on PAEXO: it is the first step of the evaluation process (many other exoskeleton studies use nonexpert participants as a first approach, e.g., [9,13,15,21,22,28$30]$ ), and a field study with factory workers is planned next. In this context, a preliminary lab study is a strong asset, if not a mandatory step, to gain access to factories for field testing. The main objective of the present study was therefore to conduct an in-depth evaluation of multiple biomechanical and physiological effects of the exoskeleton, that can hardly be investigated during field testing. Many sensors are actually difficult to use in industrial settings (e.g., force plates, VO2 mask, EMG), and field testing often resorts to less numerous and/or less accurate sensors. Among field studies on passive exoskeletons for overhead work, Moyon et al. used a simple heart rate sensor along with questionnaires [18], while Butler and Wisner monitored productivity only [11]. Gillette and Stephenson did use EMG, but solely EMG, possibly to limit the complexity and hence equipment time of the experiment [12]. Conversely, our lab study enabled a simultaneous assessment of multiple and heterogeneous effects.

A second limitation is the nature of the experimental task which focused on what PAEXO is designed for, overhead work, but ignored peripheral movements that are still needed in factory work. For instance, though participants reported that lowering the arm with PAEXO at the end of each block was not an issue, the time spent doing this movement was negligible compared to the time spent with arms raised. Yet lowering the arm might require to counter the exoskeleton upward support force. For instance, Otten et al. observed some increase in latissimus dorsi activity when lowering the arm with the exoskeleton Lucy 2.0 [14]. In a future study, such movements as well as other peripheral movements like bending or walking will be tested to ensure that the exoskeleton is not a hindrance. In addition, the level of support provided by PAEXO was chosen specifically for the task of interest. This assistance may however not be optimal in less structured tasks where other movements are involved, which is inevitable in industrial tasks. Current work is therefore directed towards developing an on-line adaptation of the level of support, either through a manual user command, or through an automatic system based on motion prediction.

Lastly, some additional measures could bring valuable information in future lab experiments. We monitored shoulder strain with EMG on $\mathrm{AD}$, the main agonist muscle active during overhead work. While this measure gives a first insight into shoulder biomechanical strain, it does not give an exhaustive picture of the effect of the exoskeleton on the whole shoulder. Other muscles also contribute to the arm elevation movement and stabilization. For instance, co-contraction of antagonist muscles is not taken into account here. Yet, Van Engelhoven et al. reported an increase in shoulder antagonist muscle activity when performing static and dynamic overhead tasks with the ShoulderX [17]. Similarly, additional muscles could be monitored to assess side effects more extensively. EMG measurement with an exoskeleton however poses an issue of sensor placement. The exoskeleton is worn directly on the body; its structure might therefore press on EMG sensors and disturb the measurement which becomes unreliable. With additional sensors, space to position them on the body in a way that is compatible with exoskeleton use becomes an issue. 


\section{CONCLUSION}

This study presented a thorough assessment of PAEXO, a lightweight upper-limb exoskeleton offering arm support during overhead work. A lab study was conducted in which non-expert participants performed an overhead pointing task, both with and without the assistance of PAEXO. Assessment of physical, physiological, and psychological aspects all suggest that PAEXO is a promising solution to reduce shoulder WMSDs among overhead workers. Working with PAEXO reduces physical strain on the shoulder as well as global physiological strain, without increasing low back strain nor degrading balance. Moreover, wearing PAEXO does not degrade task performance, though it does modify shoulder movement. Importantly, participants' opinions and attitudes are positive and in agreement with the biomechanical advantage highlighted by objective measures. Since those results were obtained with a non end-user population, future work will be directed towards a field validation of PAEXO with industrial workers in a real work situation. This field testing is made possible thanks to the in-depth evaluation that was conducted in the present study. By formalizing a list of evaluation criteria and making data available, this study also aims at facilitating comparison between different exoskeletons. If task-specific testing remains necessary before deploying an exoskeleton in a workplace, the availability of comparable results would help orient a buyer's preliminary choice.

\section{REFERENCES}

[1] E. Schneider, X. Irastorza, M. Bakhuys Roozeboom, and I. Houtman, OSH in figures: occupational safety and health in the transport sectorAn overview, Eur. Agency for Saf. and Health at Work, 2010.

[2] A. Parent-Thirion, G. Vermeylen, G. van Houten, M. Lyly-Yrjninen, I. Biletta, and J. Cabrita, Fifth European Working Conditions Survey, Eurofound, Pub. Office of the European Union, 2012.

[3] Nonfatal Occupational Injuries and Illnesses RequiringDays Away from Work, 2015, US Bureau of Labor Statistics, 2016.

[4] L. Fritzsche, J. Wegge, M. Schmauder, M. Kliegel, and K.-H. Schmidt, "Good ergonomics and team diversity reduce absenteeism and errors in car manufacturing," Ergonomics, vol. 57, no. 2, pp. 148-161, 2014.

[5] A. Luttmann, M. Jäger, B. Griefahn, G. Caffier, and F. Liebers, Preventing musculoskeletal disorders in the workplace, WHO, 2003.

[6] J. K. Sluiter, K. M. Rest, and M. H. Frings-Dresen, "Criteria document for evaluating the work-relatedness of upper-extremity musculoskeletal disorders," Scand. J. Work Environ. Health, pp. 1-102, 2001.

[7] J. R. Grieve and C. R. Dickerson, "Overhead work: Identification of evidence-based exposure guidelines," Occup. Ergon., vol. 8, no. 1, pp. 53-66, 2008.

[8] M. P. de Looze, T. Bosch, F. Krause, K. S. Stadler, and L. W. OSullivan, "Exoskeletons for industrial application and their potential effects on physical work load," Ergonomics, vol. 59, no. 5, pp. 671-681, 2016.

[9] N. Sylla, V. Bonnet, F. Colledani, and P. Fraisse, "Ergonomic contribution of able exoskeleton in automotive industry," Int. J. Ind. Ergon, vol. 44, no. 4, pp. 475-481, 2014.

[10] S. Spada, L. Ghibaudo, S. Gilotta, L. Gastaldi, and M. P. Cavatorta, "Analysis of exoskeleton introduction in industrial reality: Main issues and eaws risk assessment," in Int. Conf. Appl. Human Factors and Ergon. Springer, 2017, pp. 236-244.

[11] T. Butler and D. Wisner, "Exoskeleton technology: Making workers safer and more productive," in ASSE Professional Development Conf. and Expo. Am. Soc. of Saf. Eng., 2017.

[12] J. C. Gillette and M. L. Stephenson, "Emg assessment of a shoulder support exoskeleton during on-site job tasks," in 41st Annu. Meeting of Am. Soc. of Biomech., 2017.

[13] K. Huysamen, T. Bosch, M. de Looze, K. S. Stadler, E. Graf, and L. W O'Sullivan, "Evaluation of a passive exoskeleton for static upper limb activities," Appl. Ergon., vol. 70, pp. 148-155, 2018.
[14] B. M. Otten, R. Weidner, and A. Argubi-Wollesen, "Evaluation of a novel active exoskeleton for tasks at or above head level," IEEE Rob. Autom. Lett., vol. 3, no. 3, pp. 2408-2415, 2018.

[15] S. Kim, M. A. Nussbaum, M. I. M. Esfahani, M. M. Alemi, S. Alabdulkarim, and E. Rashedi, "Assessing the influence of a passive, upper extremity exoskeletal vest for tasks requiring arm elevation: Part iexpected effects on discomfort, shoulder muscle activity, and work task performance," Appl. Ergon., vol. 70, pp. 315-322, 2018.

[16] S. Kim, M. A. Nussbaum, M. I. M. Esfahani, M. M. Alemi, B. Jia and E. Rashedi, "Assessing the influence of a passive, upper extremity exoskeletal vest for tasks requiring arm elevation: Part ii-unexpected effects on shoulder motion, balance, and spine loading," Appl. Ergon., vol. 70, pp. 323-330, 2018.

[17] L. Van Engelhoven, N. Poon, H. Kazerooni, A. Barr, D. Rempel, and C. Harris-Adamson, "Evaluation of an adjustable support shoulder exoskeleton on static and dynamic overhead tasks," in Human Factors and Ergono. Soc. Int. Annu. Meeting, October 2018.

[18] A. Moyon, E. Poirson, and J.-F. Petiot, "Experimental study of the physical impact of a passive exoskeleton on manual sanding operations," Procedia CIRP, vol. 70, pp. 284-289, 2018.

[19] A. Voilqué, J. Masood, J. Fauroux, L. Sabourin, and O. Guezet, "Industrial exoskeleton technology: Classification, structural analysis, and structural complexity indicator," in Wearable Robotics Ass. Conf. IEEE, 2019, pp. 13-20.

[20] J. Theurel, K. Desbrosses, T. Roux, and A. Savescu, "Physiological consequences of using an upper limb exoskeleton during manual handling tasks," Appl. Ergon., vol. 67, pp. 211-217, 2018.

[21] E. B. Weston, M. Alizadeh, G. G. Knapik, X. Wang, and W. S. Marras, "Biomechanical evaluation of exoskeleton use on loading of the lumbar spine," Appl. Ergon., vol. 68, pp. 101-108, 2018.

[22] E. Rashedi, S. Kim, M. A. Nussbaum, and M. J. Agnew, "Ergonomic evaluation of a wearable assistive device for overhead work," $E r$ gonomics, vol. 57, no. 12, pp. 1864-1874, 2014.

[23] A. J. Young and D. P. Ferris, "State of the art and future directions for lower limb robotic exoskeletons," IEEE Trans. Neural Syst. Rehabil. Eng., vol. 25, no. 2, pp. 171-182, 2017.

[24] C. Nabeshima, K. Ayusawa, C. Hochberg, and E. Yoshida, "Standard performance test of wearable robots for lumbar support," IEEE Rob. Autom. Lett., vol. 3, no. 3, pp. 2182-2189, 2018.

[25] J. Bornmann, A. Kurzweg, and K. Heinrich, "Tragbare assistenzsysteme in der automobilmontage: Forschung und entwicklung innovativer orthetischer systeme zur physischen unterstützung whrend der Überkopfarbeit," 2016.

[26] VolkswagenAG, Produktion der Zukunft: Exoskelette unterstützen Mitarbeiter am Standort Bratislava, https://www.youtube.com/watch?v= AyIgUcxB0HA, accessed: 20.08.2018.

[27] S. Ivaldi, L. Fritzsche, J. Babič, F. Stulp, M. Damsgaard, B. Graimann, G. Bellusci, and F. Nori, "Anticipatory models of human movements and dynamics: the roadmap of the andy project," in Digital Hum. Model. Symp., 2017.

[28] S. Baltrusch, J. van Dieën, S. Bruijn, A. Koopman, C. van Bennekom, and H. Houdijk, "The effect of a passive trunk exoskeleton on metabolic costs during lifting and walking," Ergonomics, pp. 1-14, 2019.

[29] T. Bosch, J. van Eck, K. Knitel, and M. de Looze, "The effects of a passive exoskeleton on muscle activity, discomfort and endurance time in forward bending work," Appl. Ergon., vol. 54, pp. 212-217, 2016.

[30] B. H. Whitfield, P. A. Costigan, J. M. Stevenson, and C. L. Smallman, "Effect of an on-body ergonomic aid on oxygen consumption during a repetitive lifting task," nt. J. Ind. Ergon, vol. 44, no. 1, pp. 39-44, 2014.

[31] N. Sylla, V. Bonnet, G. Venture, N. Armande, and P. Fraisse, "Assessing neuromuscular mechanisms in human-exoskeleton interaction," in 36th Annu. Int. Conf. of IEEE Eng. Med. Biol. Soc., 2014, pp. 1210-1213.

[32] D. Ndahimana and E.-K. Kim, "Measurement methods for physical activity and energy expenditure: a review," Clinical nutrition research, vol. 6, no. 2, pp. 68-80, 2017.

[33] T. Flash and N. Hogan, "The coordination of arm movements: an experimentally confirmed mathematical model," J. Neurosci., vol. 5, no. 7, pp. 1688-1703, 1985.

[34] B. G. Borgs, "Perceived exertion and pain scales," Champaign, IL: Human Kinetics, 1998.

[35] S. G. Hart and L. E. Staveland, "Development of nasa-tlx (task load index): Results of empirical and theoretical research," in Adv. Psychol. Elsevier, 1988, vol. 52, pp. 139-183.

[36] V. Venkatesh and H. Bala, "Technology acceptance model 3 and a research agenda on interventions," Decis. Sci., vol. 39, no. 2, pp. 273 $315,2008$. 
[37] V. Venkatesh, M. G. Morris, G. B. Davis, and F. D. Davis, "User acceptance of information technology: Toward a unified view," MIS quarterly, pp. 425-478, 2003.

[38] A. Bangor, P. T. Kortum, and J. T. Miller, "An empirical evaluation of the system usability scale," Intl. Journal of Human-Computer Interaction, vol. 24, no. 6, pp. 574-594, 2008.

[39] H. J. Hermens, B. Freriks, R. Merletti, D. Stegeman, J. Blok, G. Rau, C. Disselhorst-Klug, and G. Hägg, "European recommendations for surface electromyography," Roessingh Res. and Dev., vol. 8, no. 2, 1999.

[40] L. McAtamney and E. N. Corlett, "Rula: a survey method for the investigation of work-related upper limb disorders," Appl. Ergon., vol. 24, no. 2, pp. 91-99, 1993.

[41] Comau, Exoskeleton MATE, https://www.comau.com/en/mate, accessed: 01.07.2019.

[42] P. Maurice, L. Allienne, A. Malaisé, and S. Ivaldi, "Ethical and social considerations for the introduction of human-centered technologies at work," in IEEE Workshop on Adv. Rob. and Social Impacts, 2018. 


\title{
Objective and Subjective Effects of Passive Exoskeleton on Overhead Work Supplementary Material
}

\author{
Pauline Maurice, Member IEEE, Jernej Čamernik, Daša Gorjan, Benjamin Schirrmeister, Jonas Bornmann, Luca \\ Tagliapietra, Claudia Latella, Daniele Pucci, Lars Fritzsche, Serena Ivaldi, Member IEEE, and Jan \\ Babič, Member IEEE
}

\section{EXPERIMENTAL SET-UP}

Fig. 1 depicts the positioning of the 4 start positions on the screen with respect to the participant's position.

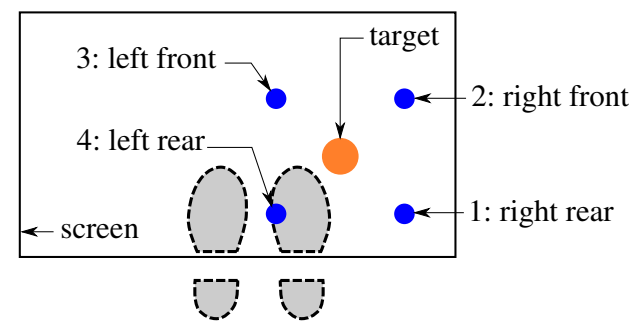

Fig. 1: Top-down schematic view of the positioning of the 4 start positions. The start positions are distributed on a circle of radius $1 / 6^{t h}$ of the participant's height and centered on the target. Note that the displayed position of the feet with respect to the target and start positions is approximate.

\section{EXPERIMENTAL DESIGN}

Fig. 2 shows the time-line of the experiment for a pair of participants, one in each of the two groups.

\section{NASA TASK LOAD INDEX}

Table I reports the detailed results of the NASA-TLX for all 12 participants (score of each construct and weights) [1]. Table II reports the average and standard deviation of NASATLX scores across all participants, for each construct and globally.

Pauline Maurice and Serena Ivaldi are with Université de Lorraine, CNRS, Inria, LORIA, F-54000 Nancy, France. Jernej Čamernik, Daša Gorjan and Jan Babič are with the Laboratory for Neuromechanics and Biorobotics, Department of Automation, Biocybernetics and Robotics, Jožef Stefan Institute, Jamova cesta 39, SI-1000 Ljubljana, Slovenia. Jernej Čamernik is also with the Jožef Stefan International Postgraduate School, Jamova cesta 39, 1000 Ljubljana, Slovenia. Benjamin Schirrmeister and Jonas Bornmann are with Ottobock SE \& Co. KGaA, Max-Näder Str. 15, 37115 Duderstadt, Germany. Luca Tagliapietra, Claudia Latella and Daniele Pucci are with the Fondazione Istituto Italiano di Tecnologia, via Morego 30, Genova, Italy. Lars Fritzsche is with imk automotive GmbH, Amselgrund 30, 09128 Chemnitz, Germany.

Pauline Maurice and Jernej Čamernik contributed equally to this work.

This work has received funding from the European Union's H2020 research and innovation programme under grant agreement No. 731540 (An.Dy).

\section{Technology Acceptance Questionnaire}

The technology acceptance questionnaire used for the exoskeleton assessment is reported in Table III. The questionnaire was designed to cover the main constructs of a Technology Acceptance Model inspired by TAM3 [2] and UTUAT [3], while trying to keep a minimal number of questions. The number of positive and negative ("reverse" in Table III) questions is equal to balance any bias introduced by the formulation of the questions. The questionnaire does not include questions for the Social Influence (SI) construct, as it is not appropriate for the nature of the experimental study in a laboratory setting with participants that are not final end-users of the system. All questions were evaluated on a scale from 0 to 10 .

Table IV reports the results of the technology acceptance questionnaire for all 12 participants. Note that the scores of the reverse questions have been inverted so that 0 corresponds to "bad opinion of the system" and 10 to "good opinion of the system" for all questions.

\section{SEMI-DiRECTEd INTERVIEW}

A semi-directed interview was conducted with each participant at the end of the experiment. Semi-directed interviews are semi-structured individual interviews where the interviewer follows a pre-determined set of questions to prompt the discussion, with the possibility to explore more in depth some answers or particular themes. A list of open questions defines the general orientation of the discussion and the specific topics that need to be discussed. The interviewer can however decide to add or remove some questions, or to change the order of the questions, depending on the answers given by the participant. Unlike a questionnaire with yes/no answers, a semi-directed interview enables participants to explain their ideas with their own words, thereby expressing more detailed opinions and providing more background to understand the motivations for their attitudes and opinions. The list of questions used to orient the semi-directed interview is given in Table V.

\section{Movement Strategy}

\section{A. Tool Movement}

Fig. 3 displays the 3D trajectory of the tool tip for all participants and both interventions WE and NE, for all 4 start 
positions and for bothl target sizes. The trajectories displayed represent only the reaching phase, i.e., they start when the tool tip leaves the start position and end when the target is first reached; the $2 \mathrm{~s}$ hold phase is not included.

Fig. 4 displays the time-series of the velocity of the tool tip for all participants and both interventions WE and NE, for all 4 start positions and for both target sizes. The time-series displayed represent only the reaching phase, i.e., they start when the tool tip leaves the start position and end when the target is first reached; the $2 \mathrm{~s}$ hold phase is not included.

\section{B. Arm Movement}

Fig. 5 displays the time-series of the shoulder and elbow joint angles of all participants for both interventions WE and $\mathrm{NE}$, for all 4 start positions and for the small target size. Fig. 6 displays the time-series of the shoulder and elbow joint angles of all participants for both interventions WE and NE, for all 4 start positions and for the large target size. The timeseries displayed represent the movement from when the tool tip leaves the start position to the end of the $2 \mathrm{~s}$ hold after the target is reached.

\section{Back Movement}

Fig. 7 and 8 display the time-series of the back joint angles (flexion, rotation and lateral bending) of all participants for both interventions WE and NE, for all 4 start positions, and for the small and large target size respectively. The timeseries displayed represent the movement from when the tool tip leaves the start position to the end of the $2 \mathrm{~s}$ hold after the target is reached. The back flexion and lateral bending are similar for both interventions WE and NE, while the back rotation rotation is slightly smaller when the exoskeleton is used (the maximum rotation angle is smaller in the WE condition, by $1.3 \mathrm{deg}$ to $3.6 \mathrm{deg}$ depending on the start position and target size).

\section{Hip Movement}

Fig. 9 displays the time-series of the right and left hip extension angles of all participants for both interventions WE and NE, for all 4 start positions and for the small target size. Fig. 10 displays the time-series of the right and left hip extension angles of all participants for both interventions WE and NE, for all 4 start positions and for the large target size. The time-series displayed represent the movement from when the tool tip leaves the start position to the end of the $2 \mathrm{~s}$ hold after the target is reached. The hip extension angles are similar for both interventions WE and NE, except the standard deviation is slightly larger without exoskeleton, for all start positions and both target sizes.

\section{REFERENCES}

[1] S. G. Hart and L. E. Staveland, "Development of nasa-tlx (task load index): Results of empirical and theoretical research," in Advances in psychology. Elsevier, 1988, vol. 52, pp. 139-183.

[2] V. Venkatesh and H. Bala, "Technology acceptance model 3 and a research agenda on interventions," Decision sciences, vol. 39, no. 2, pp. 273-315, 2008 .

[3] V. Venkatesh, M. G. Morris, G. B. Davis, and F. D. Davis, "User acceptance of information technology: Toward a unified view," MIS quarterly, pp. 425-478, 2003. 


\begin{tabular}{|c|c|c|c|c|c|c|c|c|}
\hline \multicolumn{5}{|c|}{ Participant A } & & & & \\
\hline $\mathrm{P}$ & WE & $Q-B$ & $\mathrm{NE}$ & Q - I & & & & \\
\hline $40 \mathrm{~min}$ & $\sim 12 \min$ & $15 \mathrm{~min}$ & $\sim 12 \min$ & $40 \mathrm{~min}$ & $\sim 12 \mathrm{~min}$ & $15 \mathrm{~min}$ & $\sim 12 \min$ & $15 \mathrm{~min}$ \\
\hline & & & & $\mathrm{P}$ & $\mathrm{NE}$ & $\mathrm{Q}-\mathrm{B}$ & WE & Q - I \\
\hline
\end{tabular}

Fig. 2: Time-line of the experiment for a pair of participants (one in Group A and one in Group B). The experiment includes : preparation of the participant (P), sessions with (WE) and without (NE) exoskeleton, break between sessions (B), questionnaire filling $(\mathrm{Q})$, and interview of the participant (I).

\begin{tabular}{|c|c|c|c|c|c|c|c|c|c|c|c|c|}
\hline Construct & $\mathrm{P} 01$ & $\mathrm{P} 02$ & $\mathrm{P} 03$ & $\mathrm{P} 04$ & $\mathrm{P} 05$ & P06 & $\mathrm{P} 07$ & P08 & P09 & P10 & P11 & $\mathrm{P} 12$ \\
\hline \multicolumn{13}{|c|}{ With exoskeleton } \\
\hline Mental demand & 15 & 10 & 15 & 40 & 35 & 35 & 25 & 5 & 60 & 25 & 55 & 30 \\
\hline Physical demand & 15 & 10 & 80 & 80 & 65 & 65 & 65 & 45 & 65 & 35 & 35 & 35 \\
\hline Temporal demand & 100 & 10 & 75 & 75 & 20 & 65 & 75 & 55 & 50 & 15 & 80 & 60 \\
\hline Performance & 40 & 10 & 25 & 35 & 50 & 45 & 25 & 15 & 25 & 35 & 25 & 55 \\
\hline Effort & 50 & 10 & 85 & 70 & 65 & 55 & 70 & 55 & 80 & 40 & 30 & 45 \\
\hline Frustration & 0 & 15 & 55 & 45 & 65 & 55 & 10 & 20 & 10 & 35 & 25 & 25 \\
\hline \multicolumn{13}{|c|}{ No exoskeleton } \\
\hline Mental demand & 25 & 15 & 20 & 15 & 75 & 25 & 25 & 10 & 75 & 40 & 35 & 25 \\
\hline Physical demand & 30 & 30 & 90 & 75 & 85 & 70 & 90 & 70 & 85 & 60 & 85 & 75 \\
\hline Temporal demand & 100 & 40 & 75 & 75 & 40 & 70 & 70 & 65 & 65 & 30 & 75 & 65 \\
\hline Performance & 45 & 10 & 30 & 35 & 90 & 45 & 35 & 25 & 25 & 55 & 50 & 55 \\
\hline Effort & 75 & 30 & 90 & 70 & 80 & 70 & 80 & 90 & 85 & 65 & 80 & 75 \\
\hline Frustration & 0 & 5 & 60 & 40 & 80 & 55 & 35 & 25 & 60 & 75 & 15 & 40 \\
\hline \multicolumn{13}{|c|}{ Weights } \\
\hline Mental demand & 3 & 1 & 0 & 0 & 0 & 0 & 0 & 0 & 3 & 4 & 2 & 0 \\
\hline Physical demand & 1 & 3 & 4 & 4 & 3 & 5 & 4 & 4 & 5 & 4 & 4 & 4 \\
\hline Temporal demand & 5 & 4 & 2 & 3 & 1 & 1 & 4 & 2 & 2 & 2 & 2 & 5 \\
\hline Performance & 4 & 5 & 5 & 2 & 2 & 3 & 3 & 4 & 1 & 1 & 5 & 3 \\
\hline Effort & 2 & 2 & 3 & 5 & 4 & 4 & 3 & 4 & 4 & 4 & 2 & 2 \\
\hline Frustration & 0 & 0 & 1 & 1 & 5 & 2 & 1 & 1 & 0 & 0 & 0 & 1 \\
\hline
\end{tabular}

TABLE I: Scores and weights of each construct of the NASA-TLX with and without the exoskeleton, for each participant $\mathrm{P} i$ where $i$ is the participant's ID. Scores can vary between 0 (good) and $100(\mathrm{bad})$. The sum of weights of the 6 constructs should always be 15 .

\begin{tabular}{llll}
\hline Factor & $\begin{array}{l}\text { With } \\
\text { exoskeleton }\end{array}$ & $\begin{array}{l}\text { Without } \\
\text { exoskeleton }\end{array}$ & $\begin{array}{l}\text { p-value } \\
\text { WE vs. NE }\end{array}$ \\
\hline Mental demand & $29.2 \pm 17.0$ & $32.1 \pm 21.7$ & .57 \\
Physical demand & $49.6 \pm 23.8$ & $70.4 \pm 20.9$ & $<.001$ \\
Temporal demand & $56.7 \pm 28.3$ & $64.2 \pm 19.2$ & .034 \\
Performance & $32.1 \pm 13.7$ & $41.7 \pm 20.4$ & .024 \\
Effort & $54.6 \pm 21.5$ & $74.2 \pm 15.9$ & $<.001$ \\
Frustration & $30.0 \pm 20.9$ & $40.8 \pm 26.2$ & .076 \\
\hline Global & $49.0 \pm 16.4$ & $62.3 \pm 13.8$ & $<.001$ \\
\hline
\end{tabular}

TABLE II: Average and standard deviation across participants of the NASA-TLX scores, for the two interventions WE and NE. Scores are given for the 6 factors and globally. The global score is a weighted sum of the scores of the 6 factors, computed using participant-specific weights given in Table I. Scores can vary between 0 (good) and 100 (bad). The last column displays the $\mathrm{p}$-value of the t-test comparison between the two interventions, WE and NE. 


\begin{tabular}{|c|c|c|c|c|c|}
\hline $\mathrm{N}$. & Question & $0=$ & $10=$ & Reverse & Construct \\
\hline 1 & $\begin{array}{l}\text { Wearing the exoskeleton makes me feel safe during the move- } \\
\text { ments }\end{array}$ & Not safe at all & Very safe & & TR \\
\hline 2 & I think the exoskeleton progressively improves my gestures & $\begin{array}{l}\text { Does not improve at } \\
\text { all }\end{array}$ & Improves a lot & & PU \\
\hline 3 & $\begin{array}{l}\text { I think I am loosing my autonomy when I am wearing the } \\
\text { exoskeleton }\end{array}$ & Fully autonomous & $\begin{array}{l}\text { Total loss of auton- } \\
\text { omy }\end{array}$ & $\mathrm{R}$ & ET \\
\hline 4 & I am not scared by the exoskeleton & Very scared & Not scared at all & & ATTS \\
\hline 5 & I think the exoskeleton makes me waste time & No time wasted at all & Waste too much time & $\mathrm{R}$ & PU \\
\hline 6 & $\begin{array}{l}\text { I think that the exoskeleton cannot help me doing my movements } \\
\text { in an ergonomic manner }\end{array}$ & Very helpful & Not helpful at all & $\mathrm{R}$ & TR \\
\hline 7 & I think using the exoskeleton requires some cognitive efforts & No effort at all & A lot of effort & $\mathrm{R}$ & PEOU \\
\hline 8 & $\begin{array}{l}\text { I don't feel free in my movements when I am wearing the } \\
\text { exoskeleton }\end{array}$ & Very free & Not free at all & $\mathrm{R}$ & PEOU \\
\hline 9 & I think using an exoskeleton is an important technology innovation & $\begin{array}{l}\text { Not an important in- } \\
\text { novation at all }\end{array}$ & $\begin{array}{l}\text { Very important inno- } \\
\text { vation }\end{array}$ & & ATTS \\
\hline 10 & I think you need a long training to use the exoskeleton & No training needed & Very long training & $\mathrm{R}$ & FC \\
\hline 11 & I feel less tired when using the exoskeleton for this task & Very tired & Not tired at all & & PU \\
\hline 12 & I don't like this exoskeleton & Like it a lot & Don't like it at all & $\mathrm{R}$ & ATTS \\
\hline 13 & Wearing the exoskeleton makes me feel powerful & Not powerful at all & Very powerful & & EN \\
\hline 14 & I think using the exoskeleton requires some physical effort & No effort at all & A lot of effort & $\mathrm{R}$ & PEOU \\
\hline 15 & I think the exoskeleton is easy to use & Very difficult to use & Very easy to use & & PEOU \\
\hline 16 & I think the exoskeleton is a constraint & Not a constraint at all & Strong constraint & $\mathrm{R}$ & PU \\
\hline 17 & I would prefer to use the exoskeleton if I had to do the task again & Not use it at all & Totally use it & & IU \\
\hline 18 & I trust the exoskeleton to assist my movements & No trust at all & Total trust & & TR \\
\hline 19 & I feel uncomfortable when I use the exoskeleton & Very comfortable & Very uncomfortable & $\mathrm{R}$ & ATTS \\
\hline 20 & The exoskeleton was helpful during the gestures & Not helpful at all & Very helpful & & PU \\
\hline
\end{tabular}

Legend for the constructs: PU : Perceived Usefulness - ATTS : Attitude toward the system - ET : Ethics - EN : Enjoyment - TR : Trust - PEOU : Perceived Ease of Use - FC : Facilitating Condition - IU : Intention of Use

TABLE III: Post-experimental questionnaire for technology acceptance. Questions are evaluated on a scale from 0 to 10. Reverse questions are questions formulated in a negative way regarding the acceptance of the exoskeleton.

\begin{tabular}{|c|c|c|c|c|c|c|c|c|c|c|c|c|}
\hline Question & P01 & $\mathrm{P} 02$ & $\mathrm{P} 03$ & $\mathrm{P} 04$ & P05 & P06 & P07 & P08 & P09 & P10 & $\mathrm{P} 11$ & $\mathrm{P} 12$ \\
\hline $\begin{array}{l}\text { Wearing the exoskeleton makes me feel safe during the } \\
\text { movements }\end{array}$ & 6 & 10 & 7 & 9 & 8 & 7 & 8 & 10 & 5 & 8 & 8 & 8 \\
\hline I think the exoskeleton progressively improves my gestures & 6 & 5 & 5 & 4 & 6 & 6 & 8 & 8 & 3 & 9 & 7 & 8 \\
\hline $\begin{array}{l}\text { I think I am loosing my autonomy when I am wearing the } \\
\text { exoskeleton }\end{array}$ & 7 & 4 & 8 & 8 & 8 & 4 & 9 & 8 & 8 & 8 & 5 & 7 \\
\hline I am not scared by the exoskeleton & 10 & 10 & 10 & 10 & 10 & 9 & 10 & 10 & 10 & 10 & 8 & 9 \\
\hline I think the exoskeleton makes me waste time & 9 & 8 & 10 & 6 & 10 & 8 & 9 & 10 & 10 & 7 & 9 & 9 \\
\hline $\begin{array}{l}\text { I think that the exoskeleton cannot help me doing my move- } \\
\text { ments in an ergonomic manner }\end{array}$ & 10 & 8 & 7 & 7 & 8 & 7 & 8 & 9 & 8 & 9 & 6 & 8 \\
\hline I think using the exoskeleton requires some cognitive efforts & 7 & 4 & 9 & 8 & 10 & 7 & 9 & 8 & 10 & 7 & 7 & 8 \\
\hline $\begin{array}{l}\text { I don't feel free in my movements when I am wearing the } \\
\text { exoskeleton }\end{array}$ & 9 & 7 & 7 & 4 & 8 & 3 & 8 & 8 & 7 & 8 & 6 & 7 \\
\hline $\begin{array}{l}\text { I think using an exoskeleton is an important technology } \\
\text { innovation }\end{array}$ & 10 & 7 & 6 & 7 & 9 & 7 & 8 & 5 & 9 & 9 & 9 & 9 \\
\hline I think you need a long training to use the exoskeleton & 10 & 9 & 9 & 8 & 10 & 5 & 10 & 10 & 7 & 9 & 9 & 8 \\
\hline I feel less tired when using the exoskeleton for this task & 8 & 8 & 6 & 6 & 7 & 7 & 8 & 7 & 8 & 9 & 7 & 6 \\
\hline I don't like this exoskeleton & 10 & 8 & 10 & 7 & 10 & 7 & 10 & 7 & 7 & 10 & 7 & 7 \\
\hline Wearing the exoskeleton makes me feel powerful & 8 & 5 & 7 & 4 & 9 & 6 & 8 & 7 & 5 & 6 & 7 & 7 \\
\hline I think using the exoskeleton requires some physical effort & 5 & 9 & 9 & 6 & 9 & 4 & 8 & 5 & 4 & 8 & 7 & 7 \\
\hline I think the exoskeleton is easy to use & 10 & 9 & 10 & 8 & 10 & 5 & 10 & 9 & 8 & 8 & 10 & 8 \\
\hline I think the exoskeleton is a constraint & 9 & 5 & 9 & 4 & 8 & 3 & 10 & 8 & 4 & 8 & 4 & 7 \\
\hline $\begin{array}{l}\text { I would prefer to use the exoskeleton if I had to do the task } \\
\text { again }\end{array}$ & 2 & 10 & 10 & 7 & 10 & 8 & 10 & 10 & 10 & 10 & 10 & 9 \\
\hline I trust the exoskeleton to assist my movements & 9 & 9 & 9 & 7 & 9 & 7 & 10 & 10 & 8 & 8 & 8 & 8 \\
\hline I feel uncomfortable when I use the exoskeleton & 9 & 8 & 8 & 6 & 4 & 5 & 8 & 9 & 6 & 9 & 3 & 7 \\
\hline The exoskeleton was helpful during the gestures & 7 & 9 & 8 & 7 & 8 & 6 & 8 & 9 & 10 & 9 & 9 & 8 \\
\hline
\end{tabular}

TABLE IV: Results of the questionnaire for technology acceptance for each participant $\mathrm{P} i$ where $i$ is the participant's ID. Scores can vary between 0 and 10 . Scores of reverse questions have been inverted so that 0 corresponds to "bad opinion of the system" and 10 to "good opinion of the system" for all questions. 
1) Overall, how did you feel performing the task with and without the exoskeleton?

- Difference between with and without?

- General feeling without: difficult? Physically fatiguing?

- General feeling with the exoskeleton: more/less effort doing the task? Physically and mentally?

- General feeling: was its support providing you a relief?

2) How did you feel when you first wear the exoskeleton?

- Sensation of general comfort / discomfort / neutral?

- How does the contact between the exoskeleton and your body make you feel?

- Impression that your body is constrained?

- How about the idea of being "augmented"? "Empowered"? "Iron man"?

- Were the instructions enough?

- Did you feel safe?

- Did you have any fears or concerns? Of what?

- Overall, positive or negative impression at first?

3) How did you feel during the first trials?

- Sensation of general comfort / discomfort? Any change in time?

- Did you feel increased temperature (feeling hotter or colder)?

- How were your movements? Were some movements specifically easier/harder than without the exoskeleton?

- Did you have the impression that you were constrained?

- Did you have the impression that you were making better movements with time?

- Did you feel safe? Safer or less than at the beginning?

- Any fears, concerns?

- Overall, was the sensation of wearing and using the exoskeleton becoming better or worse?

4) How did you feel towards the end of the trials?

- Did you feel that you were getting used to the exoskeleton?

- How about the level of assistance of the exoskeleton: correct/not enough/too much?

- Did you feel that you had learned how to use it?

- Did you feel the exoskeleton was specifically helpful/annoying during specific movements or parts of the task?

- Did you feel safe? Safer or less than at the beginning?

- Any fears, concerns?

- Overall, was the sensation of wearing and using the exoskeleton better or worse than the initial one (the first impression)?

- Did you feel pain in the neck during the task?

5) Overall, what is your general evaluation at the end of the experiment?

- Do you feel the support of the exoskeleton a relief for you or not?

- Is it comfortable to wear? Comfortable to use?

- Does it allow you to move freely or do you feel constrained?

- Overall, do you feel positive/neutral/negative towards the system?

- Would you use it again for overhead work? Would you recommend it to use for workers that perform a lot of overhead work?

6) What are, in your opinion, the elements to improve in using the exoskeleton?

- Design

- Weight

- Temperature

- Safety

TABLE V: List of questions used to orient the semi-directed interview. 


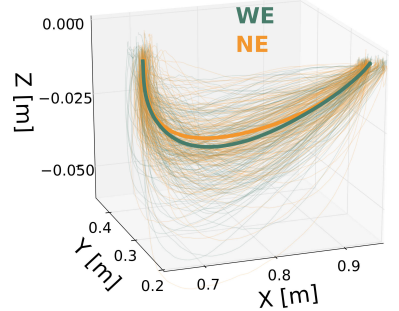

(a) Start position on the right front side of the participants, small target.

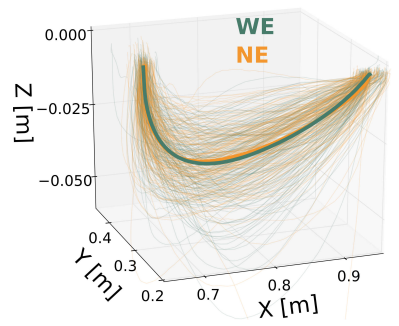

(e) Start position on the right front side of the participants, large target.

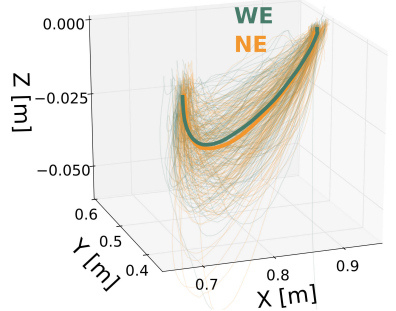

(b) Start position on the right rear side of the participants, small target.

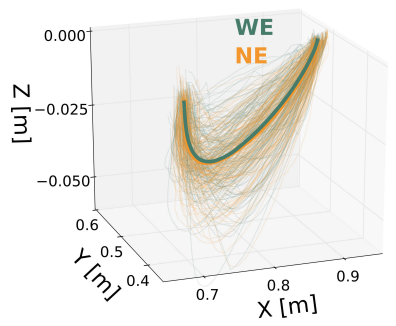

(f) Start position on the right rear side of the participants, large target.

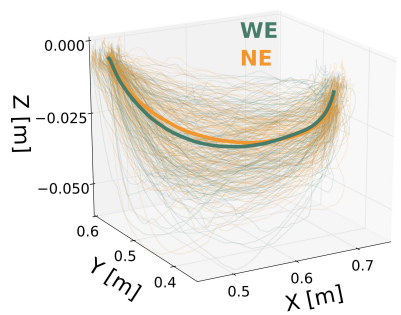

(c) Start position on the left rear side of the participants, small target.

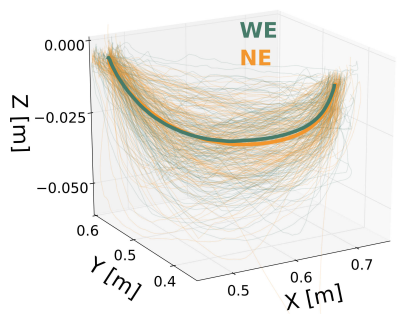

(g) Start position on the left rear side of the participants, large target.

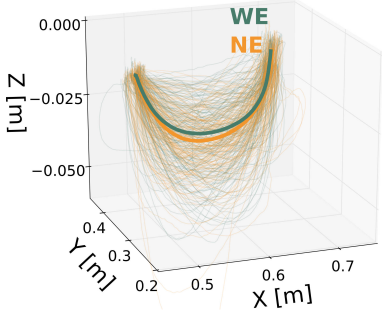

(d) Start position on the left front side of the participants, small target.

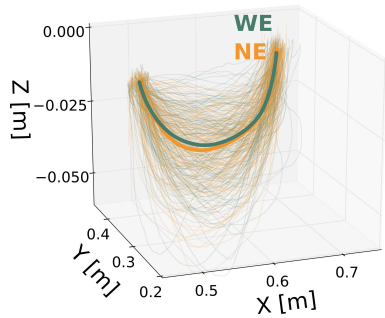

(h) Start position on the left front side of the participants, large target.

Fig. 3: 3D trajectory of the tool tip of all participants, for the two interventions WE and NE, for trials corresponding to the small target size (top) and the large target size (bottom). The trajectories displayed here represent only the reaching phase, i.e., they start when the tool tip leaves the start position ( $0 \%$ of movement time) and end when the target is first reached (100\% of movement time); the $2 \mathrm{~s}$ hold phase is not included. Thin lines, bold lines and shaded areas represent respectively individual trials, average, and standard deviation profiles (all participants, all blocks). 


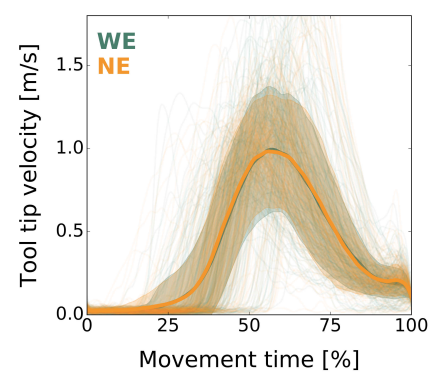

(a) Start position on the right front side of the participants, small target.

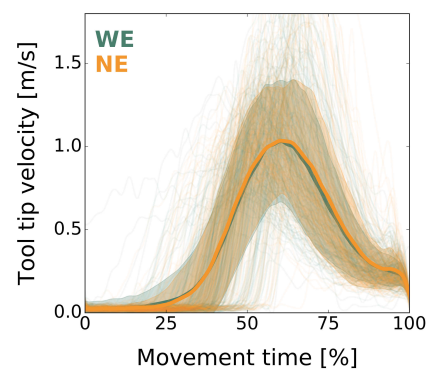

(e) Start position on the right front side of the participants, large target.

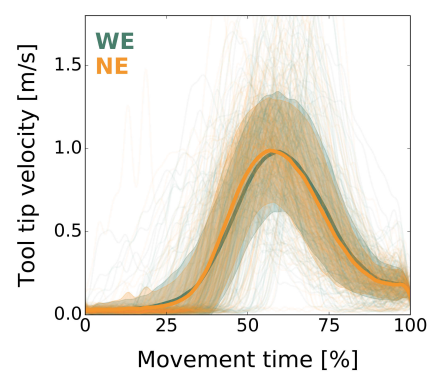

(b) Start position on the right rear side of the participants, small target.

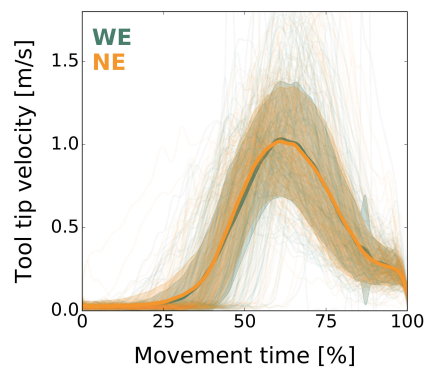

(f) Start position on the right rear side of the participants, large target.

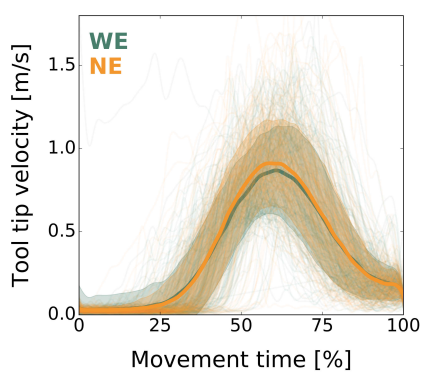

(c) Start position on the left rear side of the participants, small target.

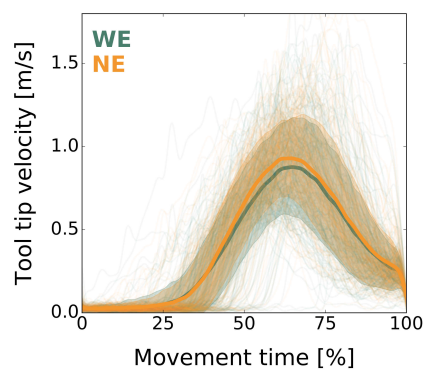

(g) Start position on the left rear side of the participants, large target.

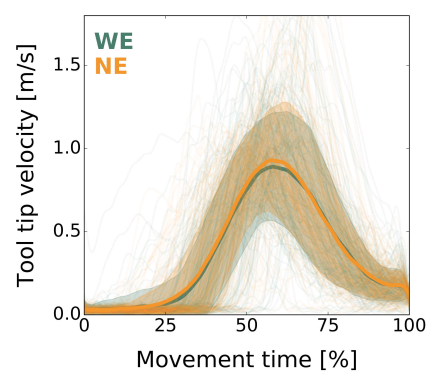

(d) Start position on the left front side of the participants, small target.

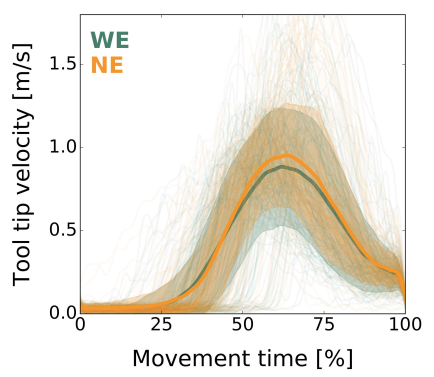

(h) Start position on the left front side of the participants, large target.

Fig. 4: Time-series of the velocity of the tool tip of all participants, for the two interventions WE and NE, for trials corresponding to the small target size (top) and the large target size (bottom). The time-series displayed here represent only the reaching phase, i.e., they start when the tool tip leaves the start position $(0 \%$ of movement time) and end when the target is first reached (100\% of movement time); the $2 \mathrm{~s}$ hold phase is not included. Thin lines, bold lines and shaded areas represent respectively individual trials, average, and standard deviation profiles (all participants, all blocks). 

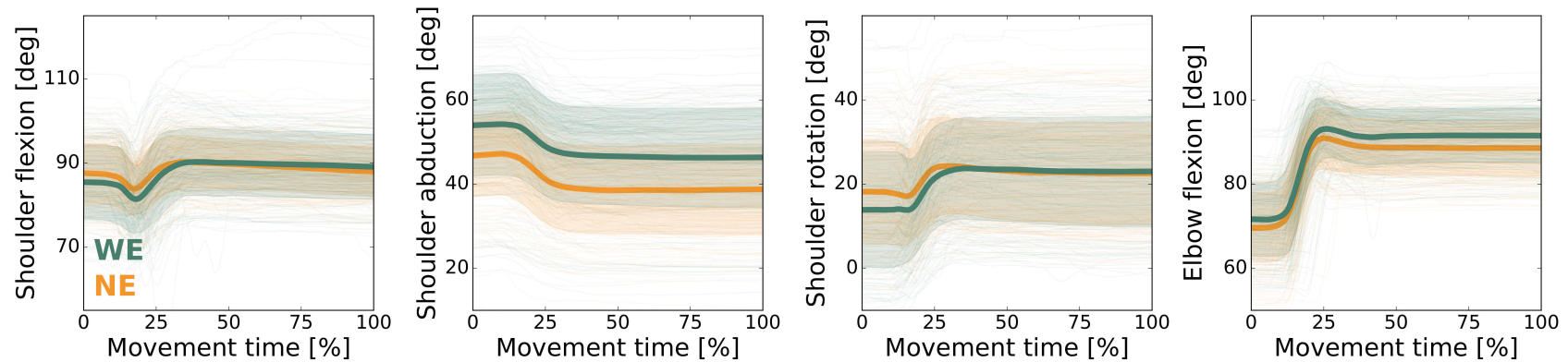

(a) Start position on the right front side of the participants, small target.
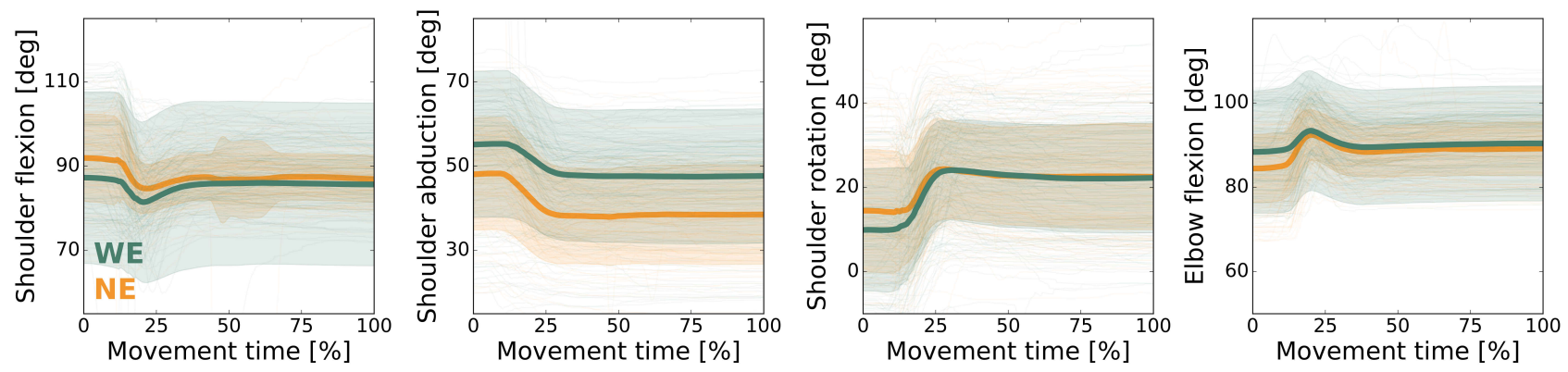

(b) Start position on the right rear side of the participants, small target.
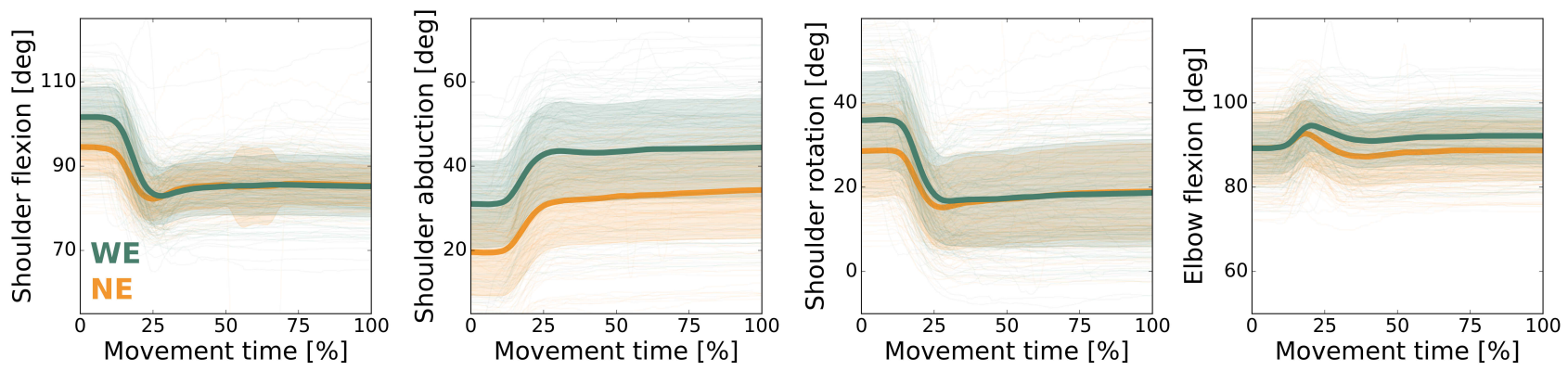

(c) Start position on the left rear side of the participants, small target.
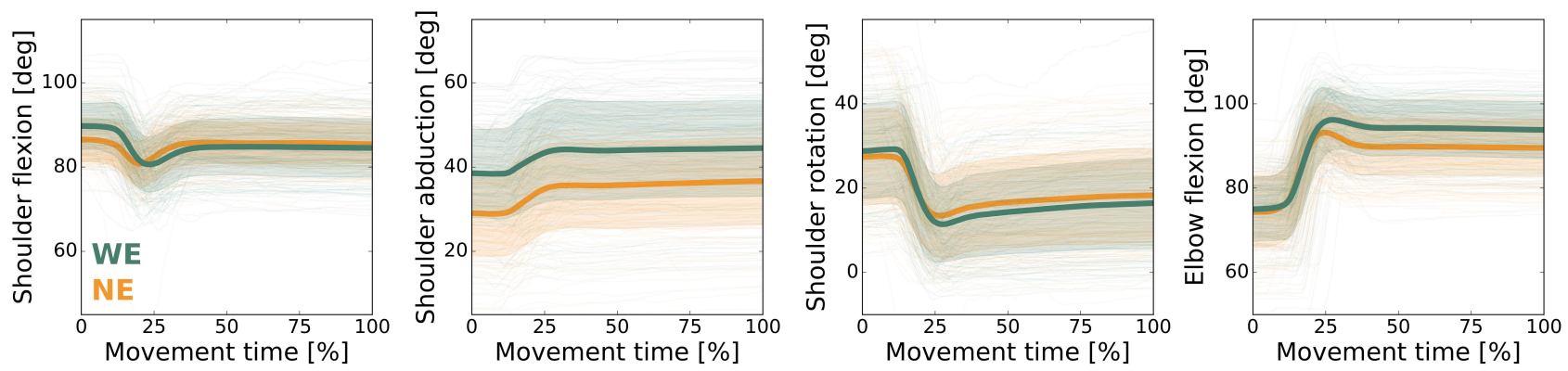

(d) Start position on the left front side of the participants, small target.

Fig. 5: Time-series of the right shoulder and elbow joints of all participants, for the two interventions WE and NE, for trials corresponding to the small target size. The time-series displayed here represent the movement from when the tool tip leaves the start position ( $0 \%$ of movement time) to the end of the $2 \mathrm{~s}$ hold after the target is reached (100\% of movement time). Thin lines, bold lines and shaded areas represent respectively individual trials, average, and standard deviation profiles (all participants, all blocks). 

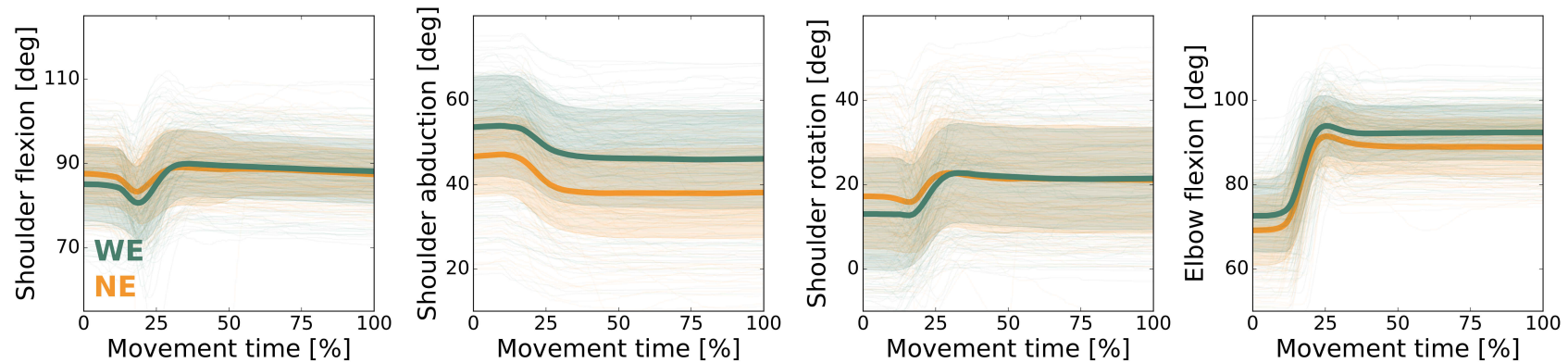

(a) Start position on the right front side of the participants, large target.
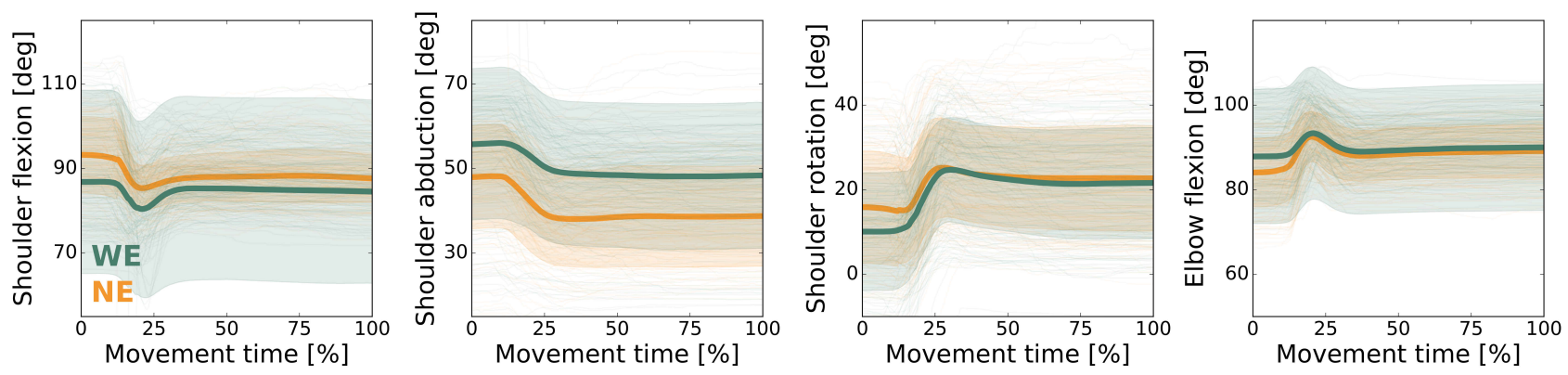

(b) Start position on the right rear side of the participants, large target.
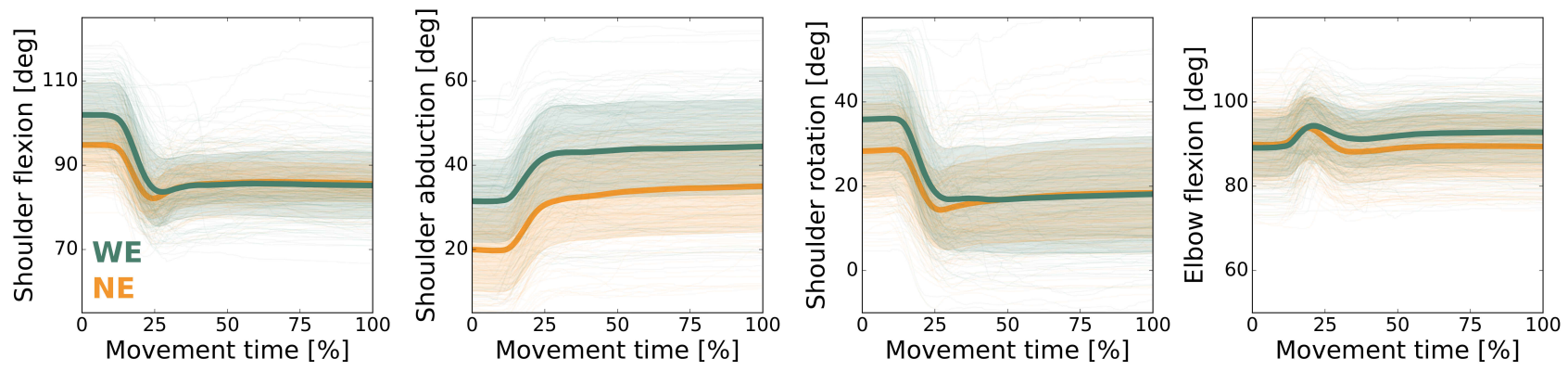

(c) Start position on the left rear side of the participants, large target.
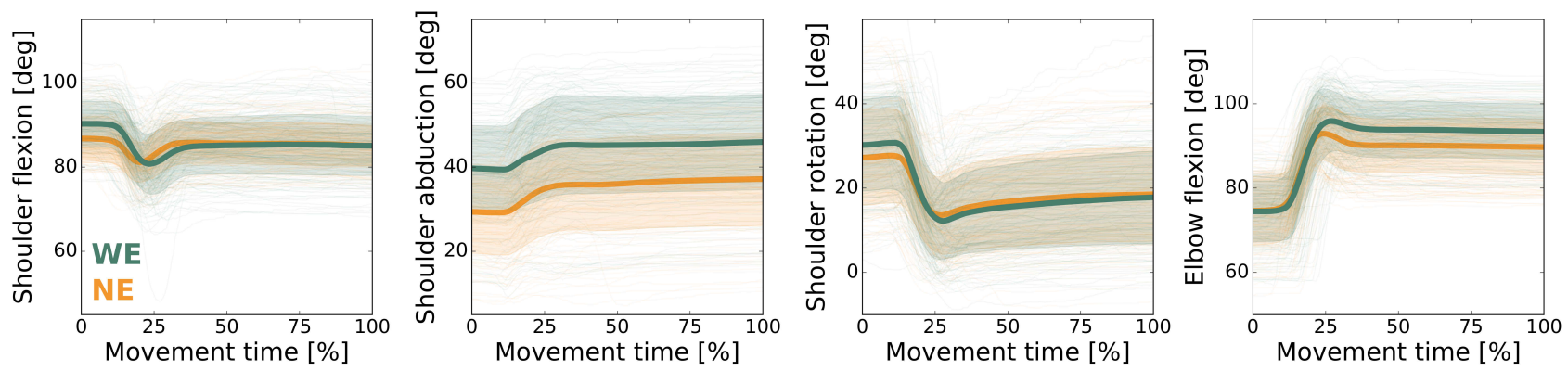

(d) Start position on the left front side of the participants, large target.

Fig. 6: Time-series of the right shoulder and elbow joints of all participants, for the two interventions WE and NE, for trials corresponding to the large target size. The time-series displayed here represent the movement from when the tool tip leaves the start position ( $0 \%$ of movement time) to the end of the $2 \mathrm{~s}$ hold after the target is reached ( $100 \%$ of movement time). Thin lines, bold lines and shaded areas represent respectively individual trials, average, and standard deviation profiles (all participants, all blocks). 

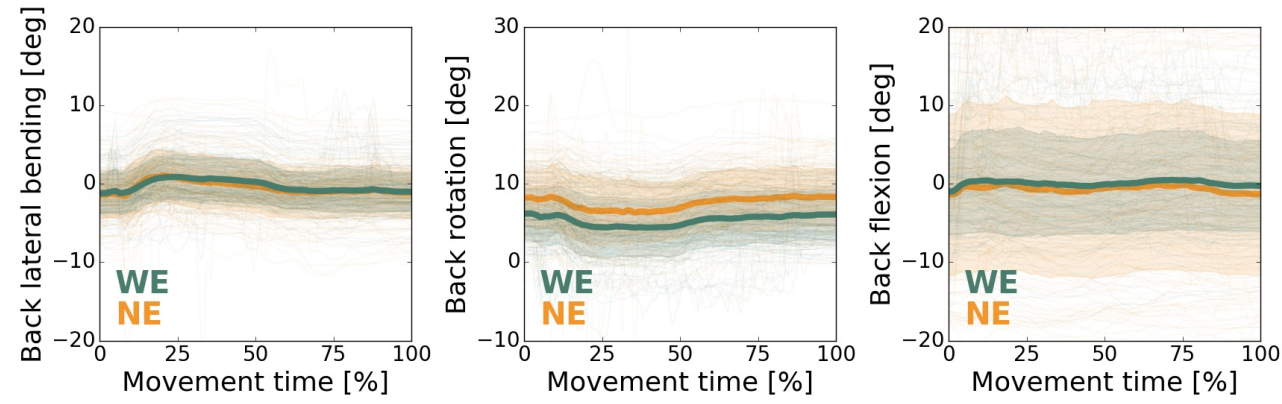

(a) Start position on the right front side of the participants, small target.
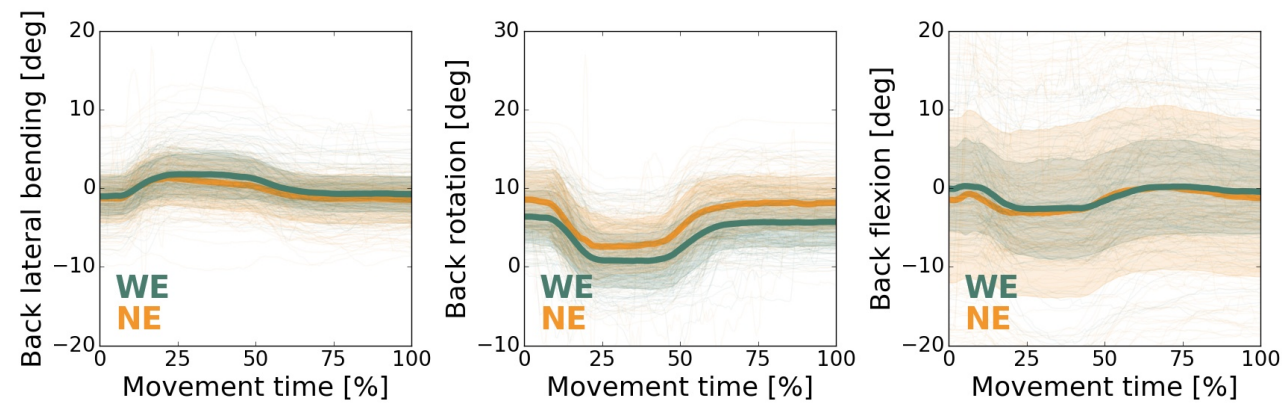

(b) Start position on the right rear side of the participants, small target.
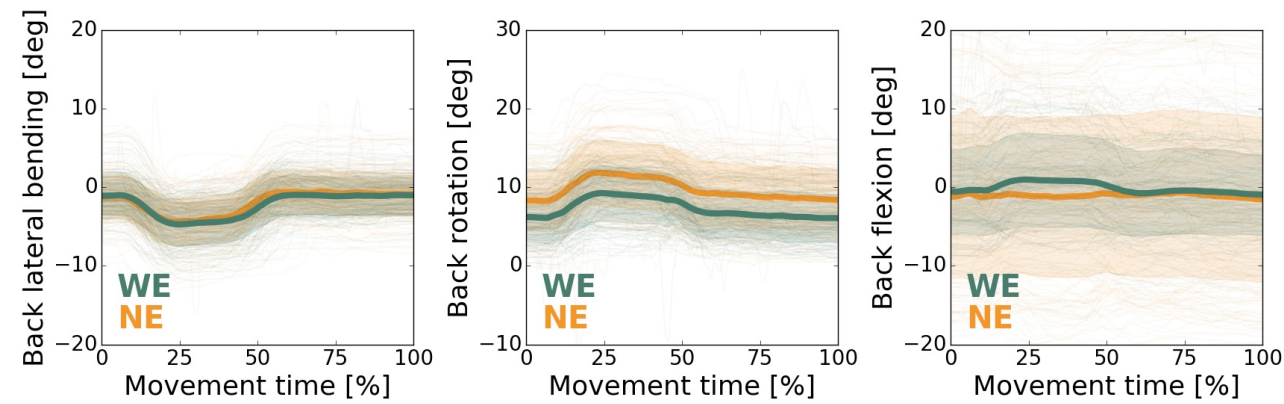

(c) Start position on the left rear side of the participants, small target.
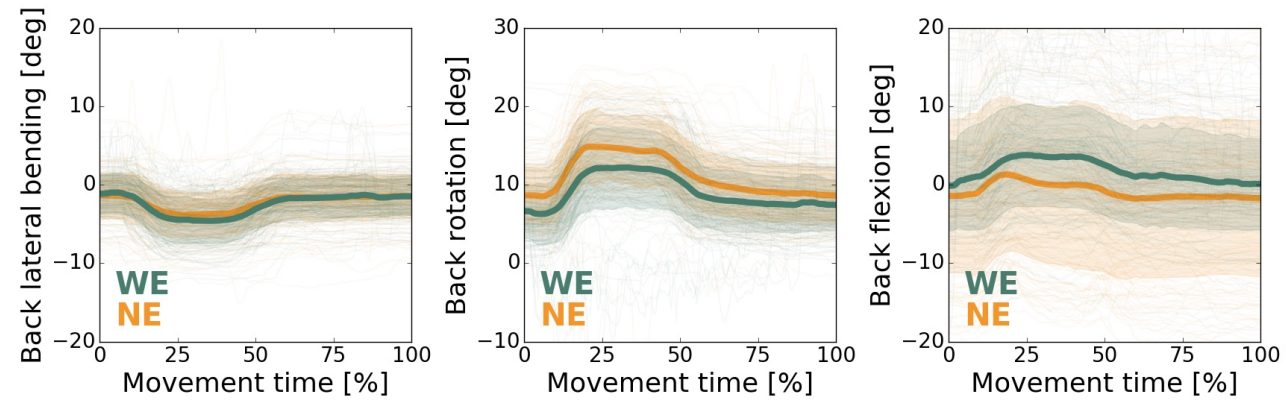

(d) Start position on the left front side of the participants, small target.

Fig. 7: Time-series of the back joint angles of all participants, for the two interventions WE and NE, for trials corresponding to the small target size. The time-series displayed here represent the movement from when the tool tip leaves the start position ( $0 \%$ of movement time) to the end of the $2 \mathrm{~s}$ hold after the target is reached (100\% of movement time). Thin lines, bold lines and shaded areas represent respectively individual trials, average, and standard deviation profiles (all participants, all blocks). 

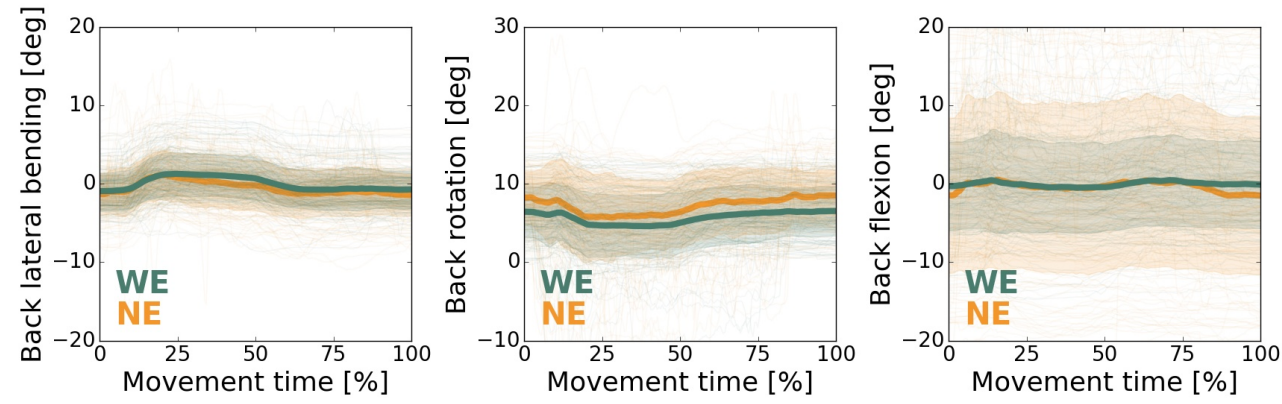

(a) Start position on the right front side of the participants, large target.
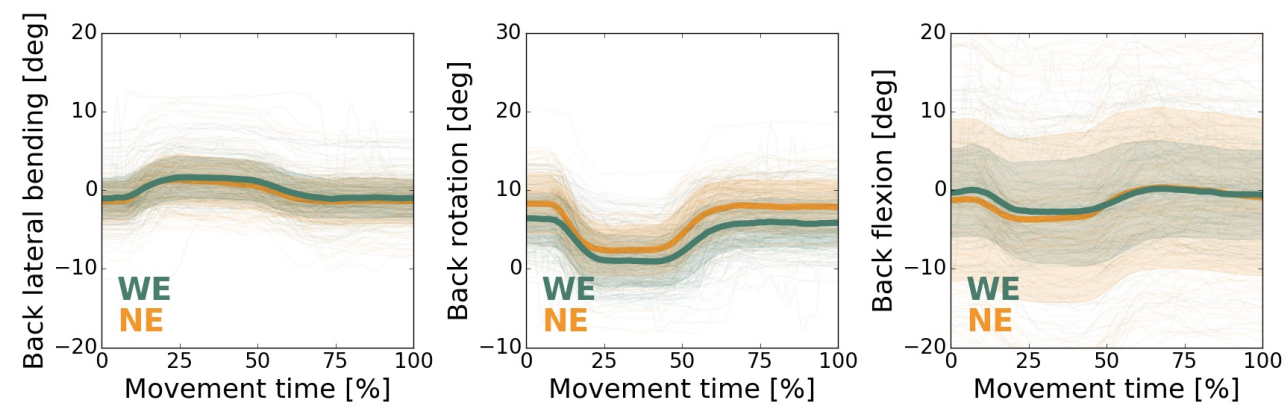

(b) Start position on the right rear side of the participants, large target.
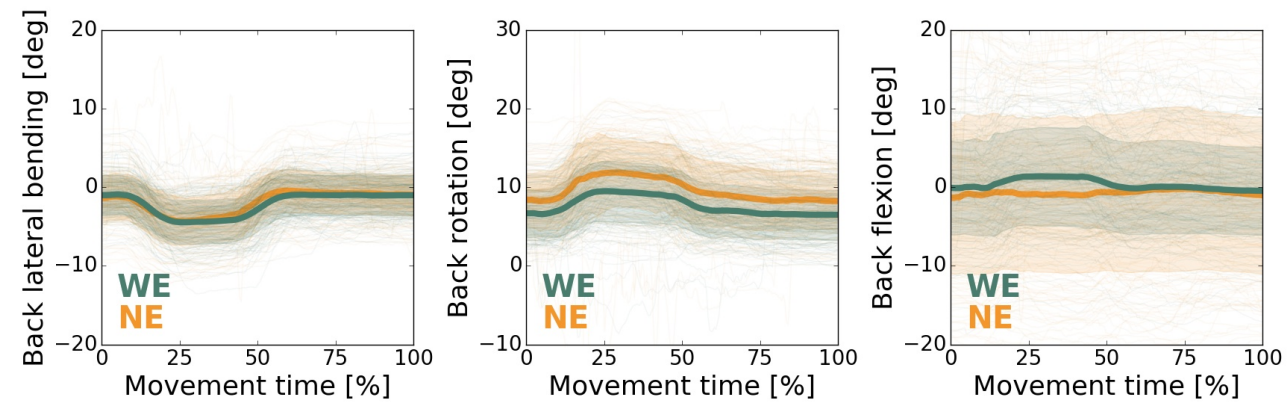

(c) Start position on the left rear side of the participants, large target.
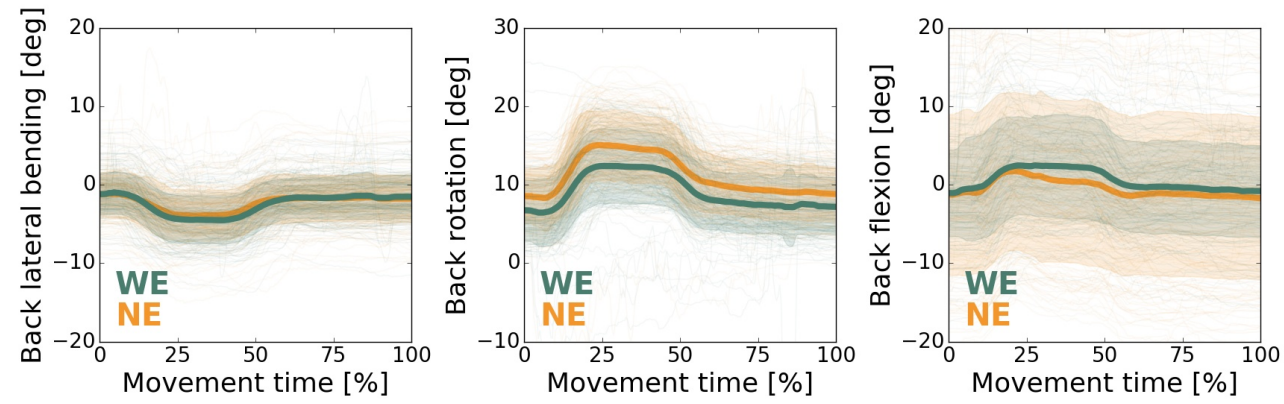

(d) Start position on the left front side of the participants, large target.

Fig. 8: Time-series of the back joint angles of all participants, for the two interventions WE and NE, for trials corresponding to the large target size. The time-series displayed here represent the movement from when the tool tip leaves the start position ( $0 \%$ of movement time) to the end of the $2 \mathrm{~s}$ hold after the target is reached (100\% of movement time). Thin lines, bold lines and shaded areas represent respectively individual trials, average, and standard deviation profiles (all participants, all blocks). 

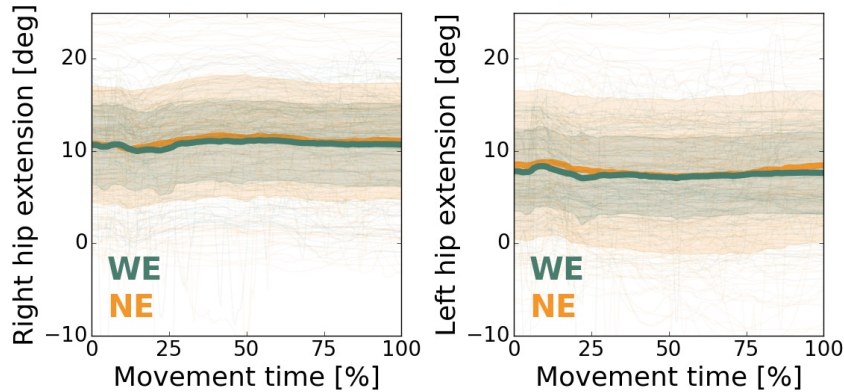

(a) Start position on the right front side of the participants, small target.
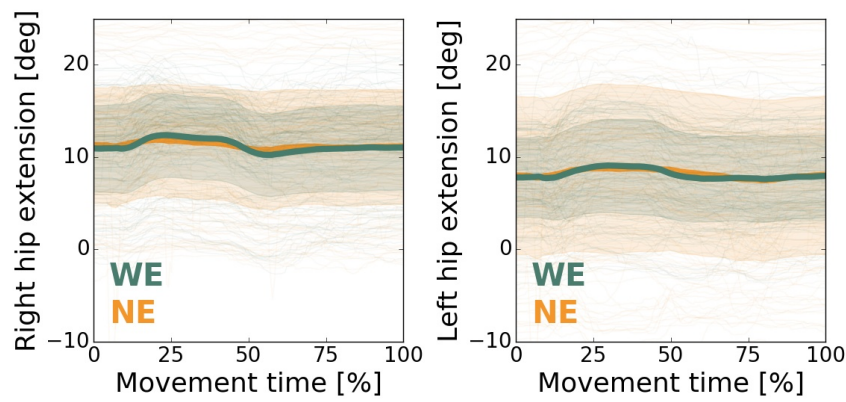

(c) Start position on the left rear side of the participants, small target.
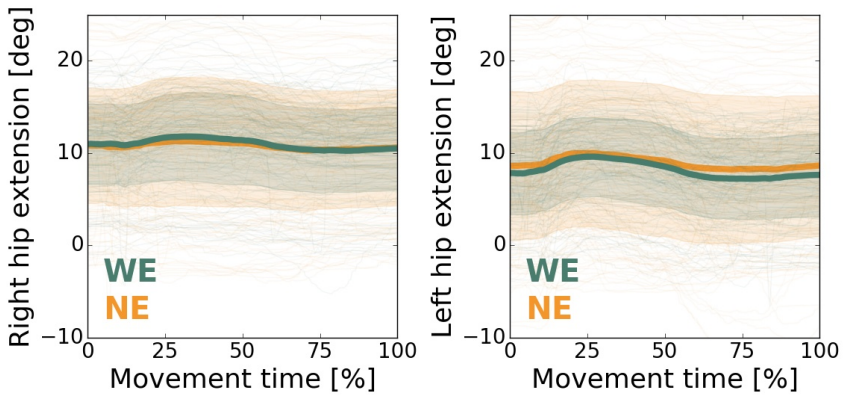

(b) Start position on the right rear side of the participants, small target.
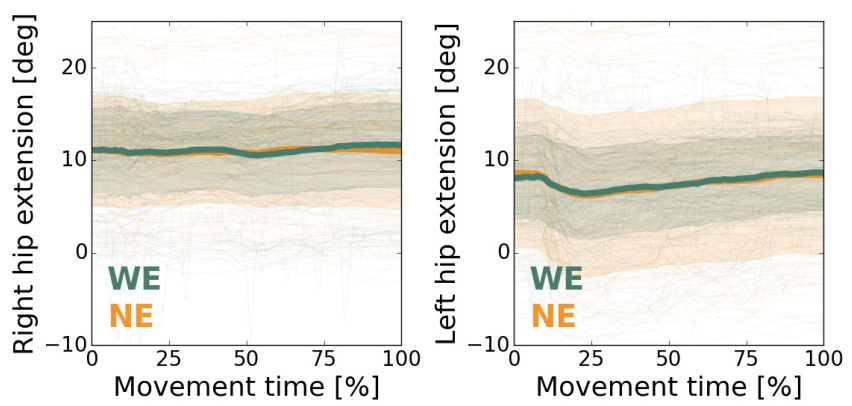

(d) Start position on the left front side of the participants, small target.

Fig. 9: Time-series of the right and left hip extension of all participants, for the two interventions WE and NE, for trials corresponding to the small target size. The time-series displayed here represent the movement from when the tool tip leaves the start position ( $0 \%$ of movement time) to the end of the $2 \mathrm{~s}$ hold after the target is reached (100\% of movement time). Thin lines, bold lines and shaded areas represent respectively individual trials, average, and standard deviation profiles (all participants, all blocks). 

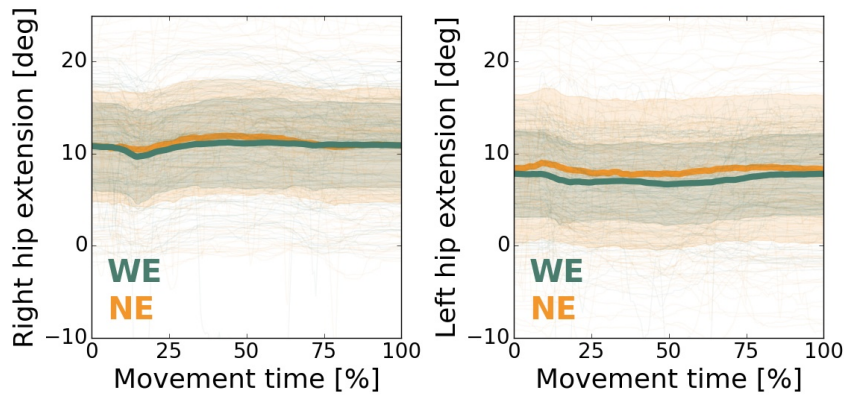

(a) Start position on the right front side of the participants, large target.
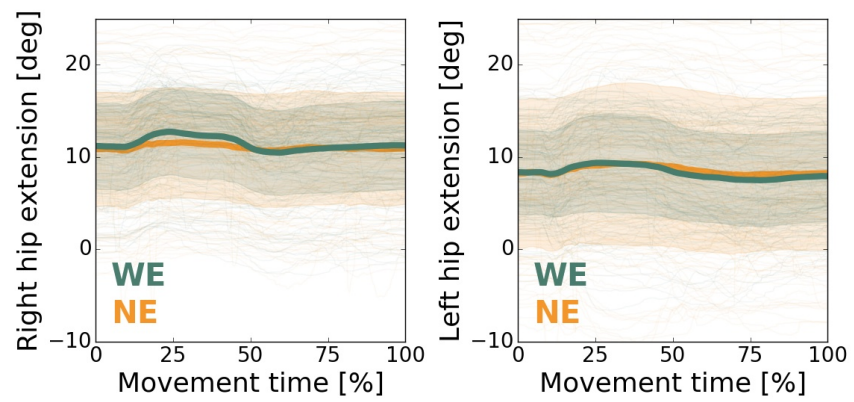

(c) Start position on the left rear side of the participants, large target.
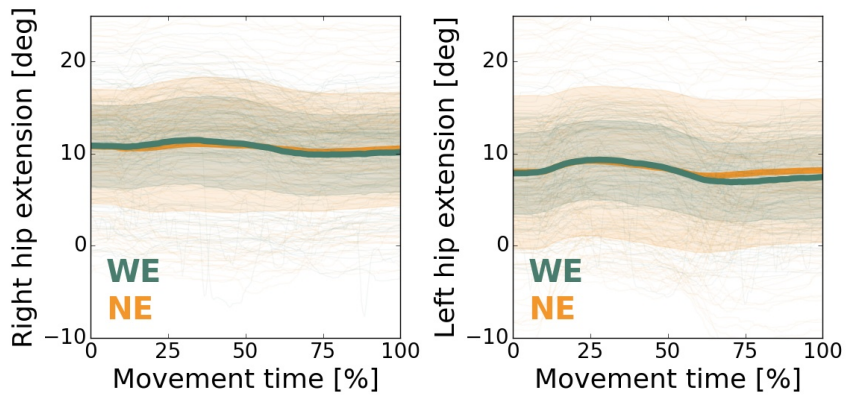

(b) Start position on the right rear side of the participants, large target.
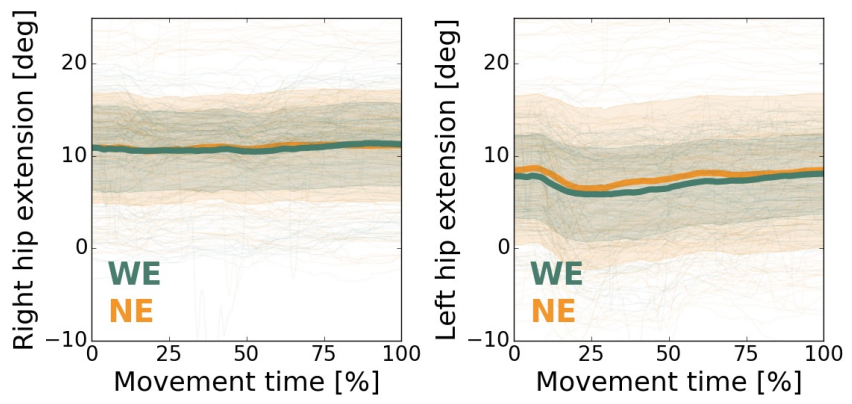

(d) Start position on the left front side of the participants, large target.

Fig. 10: Time-series of the right and left hip extension angles of all participants, for the two interventions WE and NE, for trials corresponding to the large target size. The time-series displayed here represent the movement from when the tool tip leaves the start position ( $0 \%$ of movement time) to the end of the $2 \mathrm{~s}$ hold after the target is reached (100\% of movement time). Thin lines, bold lines and shaded areas represent respectively individual trials, average, and standard deviation profiles (all participants, all blocks). 